\title{
MORPHOMETRIC ANALYSIS \\ TO EXTRAPOLATE GEOECOLOGICAL POTENTIAL OF THE RIVERS IN THE “IRON GATES” NATURAL PARK (BANAT, ROMANIA)
}

Cristian TETELEA*

* Invisible Nature, Constantin Rădulescu Motru Street 22, Bucharest, Romania, RO-040361, cristiantetelea@gmail.com geoecology.

KEYWORDS: Romania, "Iron Gates” Natural Park, rivers, morphometric analyses,

\section{ABSTRACT}

The present study is based on morphometric analysis of watersheds from the "Iron Gates” Natural Park. Morphometric parameters of a river basin are influenced by a series of biotic and abiotic factors. This aspect makes the morphometric analysis very useful in describing the river systems and offers an image of all the interactions and processes that define the geoecological potential of running waters ecosystems. The parameters used in morphometric analysis are a good alternative for understanding the underlying geoecological factors at watershed scale, and especially where the necessary data on soil, lithology, geomorphology, vegetation and other are scarce.

ZUSAMMENFASSUNG: Morphometrische Analyse zur Extrapolation des geoökologischen Potentials der Flüsse im Naturpark Eisernes Tor (Banat, Rumänien).

Die vorliegende Studie beruht auf einer morphometrischen Analyse der Einzugsgebiete und Wasserscheiden im Naturpark Eisernes Tor. Die morphometrischen Parameter eines Einzugsgebietes sind beeinflusst durch eine Reihe biotischer und abiotischer Faktoren. Dieser Aspekt erweist sich als sehr nützlich für die Beschreibung der Fluss-Systeme und ermöglicht einen Überblick über alle Zwischenbeziehungen und Prozesse, die das geoökologische Potential von Fließgewässerökosystemen definieren. Die für die morphometrische Analyse verwendeten Parameter stellen eine gute Alternative zum Verständnis der zugrunde gelegten geoökologischen Faktoren auf Einzugsgebietsebene dar, vor allerm dort, wo die notwendigen Daten zu Böden, Lithologie, Geomorphologie, Vegetation und andere spärlich sind.

REZUMAT: Analiza parametrilor morfometrici utilizați în evaluarea potențialului geoecologic al râurilor din Parcul Natural Porţile de Fier (Banat, România).

Studiul de față se bazează pe analiza parametrilor morfometrici ai bazinelor hidrografice și rețelei de râuri din Parcul Natural Porțile de Fier. Parametrii morfometrici ai unui bazin hidrografic sunt influențaţi de o serie de factori biotici și abiotici. Acest fapt face ca analiza morfometrică să fie utilă în descrierea sistemului râului oferind o imagine a interacțiunilor proceselor care definesc potențialul geoecologic al ecosistemelor apelor curgătoare. Parametrii utilizați în analiza morfometrică constituie o alternativă bună pentru înțelegerea factorilor geoecologici la nivelul bazinului, în special acolo unde lipsesc datele despre sol, litologie, geomorfologie, vegetație și altele. 


\section{INTRODUCTION}

The fundamental differences between the sciences that focus on aquatic ecosystem have imposed different approaches and perceptions of these ecosystems in general, and of running waters in particular. Some researchers perceive the rivers from the point of view of benthic invertebrates or fish on a given river or physico-chemical parameters on the condition the existence of this community of organisms (Curtean et al., 1999; Manko, 2008; Trichkova, 2009). On the other side, hydrologists perceive the same river in terms of water velocity, roughness of river bed, turbidity, gravel size, etc., and simplify these physical dimensions of the river to different relations that are determinant for the evaluation of potential river changes to the river flow dynamic. None of these approaches is superior, each represents a fraction of what should be acknowledged of the river ecosystems. It is essential to be informed of the hydrological, geological, morphological and vegetational setting of a river. A geoecological approach to rivers looks at a way of integrating the information from different sciences into a "complete image" that best defines the river system in a watershed (Tetelea, 2005).

Hydrologic and geomorphic processes appear within the basin, and morphometric characterization at the basin scale offer data regarding formation and development of land surface processes and thus furnish a holistic insight into the hydrologic comportment of a basin (Farrukh et al., 2013). Morphometric analysis offers a good alternative to understand the underlying geoecological factors at basin scale where other data on soil, lithology, geomorphology, vegetation, and so forth is scarce. Moreover, some of the morphometric parameters such as bifurcation ratio and circularity ratio are input parameters in the hydrograph analysis and evaluation of surface water potential of a specific area (Farrukh et al., 2013). In this context the morphometric analysis represent a better analysis of hydrologic behaviour of study area where gauge stations are missing.

The aquatic ecosystems' research in the "Iron Gates" Natural Park has been focused to a large extent on the Danube water body and less to the tributary rivers. Moreover, the geoecological approach of aquatic ecosystems and especially of the river systems is sustained by the fact that these ecosystems have not been studied since the establishment of the "Iron Gates" Dam in beginning of '70s. These were the main arguments to study the potential of the river system from the "Iron Gates" Natural Park from a geoecological perspective.

The proposed approach offers the opportunity to obtain practical and applied results necessary for the management of both the water body, as defined in the EU Water Framework Directive, and nature conservation. The scope of the study is to promote those morphometric elements of the river system that have a direct projection in the structure of the river network and functionality of river ecosystems.

\section{MATERIAL AND METHODS}

The present study is based on morphometric analysis of river basins and drainage network that reflects the geoecological potential of rivers from the "Iron Gates" Natural Park.

Morphometry is the measurement and mathematical analysis of the configuration of earth's surface, shape, patterns, and dimension of landforms. The understanding of evolution and behaviour of drainage patterns through quantitative methods if fundamental in catchment characteristics determination. Because of the inter-relationships between factors, one (usually the one most easily measured) can often serve as a surrogate for others. The selected factors can be used in the prediction of a catchment's hydrologic response to rainfall and for distinguishing one catchment from another for comparison or classification. 
The complex interactions between all living communities and their environmental factors are called biogeoecology (Troll, 1969/1971). The biogeoecology recognizes the importance of geographic relations (space, altitude, scale, and exposure, etc.) in modelling the complex interactions between biotic and abiotic factors of the landscape (Tetelea, 2005). The geosystem represents the smallest homogeneous unit that can be visible at the chosen scale. The basic unit in hydrological analyses is the watershed, an area of surface whose major runoff is conveyed to a single outlet. The watershed incorporates all the interactions and processes that shape the landscape. The forming elements of the landscape and their role in the dynamics and potential of running waters are determined by a hierarchical control of processes and interactions within a geosystem (Fig. 1).

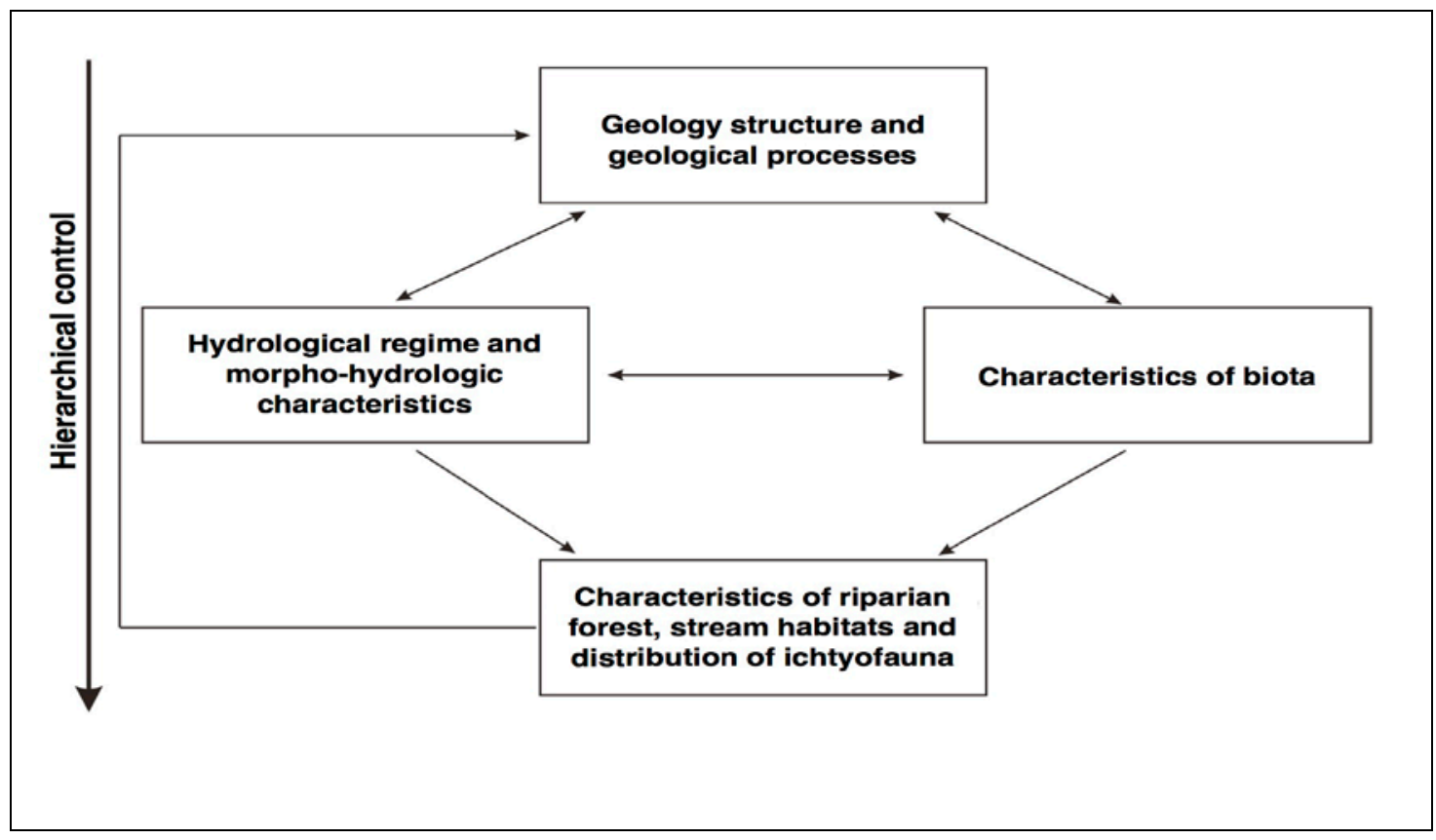

Figure 1: Hierarchical control of fundamental interactions in river systems (Naiman and Bilby, 2001).

This control can be shown through morphometric analysis of watersheds. The identification of watershed geomorphology and physical processes forms the basis for understanding the spatial extent of the riparian forest (Naiman and Bilby, 2001) as well as stream habitats, their quality, and the diversity and distribution of ichthyofauna.

River network in the "Iron Gates" Natural Park (Fig. 2) was generated in ArcGIS software from topographical maps at 1:25,000 and using a digital elevation model (DEM). Boundaries of 63 watersheds were delineated by defining the entire area contributing to flow at an outlet based on knowledge of topography. Area of watershed and perimeter were calculated based on the geometry of the derived watershed polygons. Drainage network was derived for each watershed from the "Iron Gates" Natural Park and all rivers were characterized based on stream order, flow type (perennial, intermittent), stream length, altitude at spring, and altitude at confluence. 
Streams were classified using the system proposed by Sthraler in 1952 improved by Zăvoianu in 1978. The river network delineated from the topographical maps was extended with stream segments, which are usually not shown but can be derived from the contour lines. These segments have an intermittent character and were considered in the morphometric analysis. The river network of all the watersheds that are not entirely included in the limits of the "Iron Gates" Natural Park (e.g. Bahna, Mraconia, Berzasca, and Orevița) was fully derived in order to correctly establish all the relations of the proposed morphometric analysis.

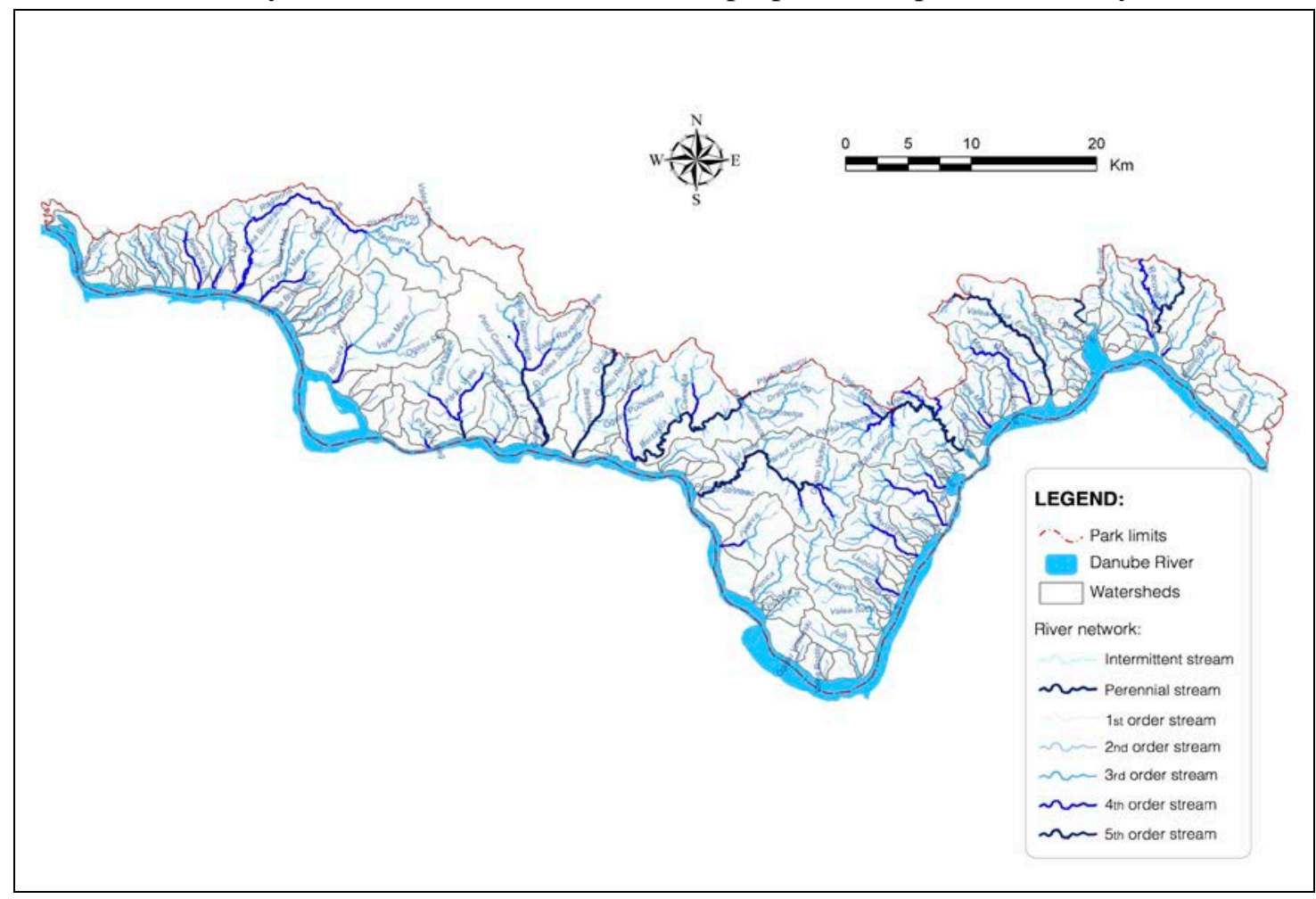

Figure 2: The hydrography of the "Iron Gates” Natural Park.

In the present study morphometric analysis of the parameters; namely, watershed surface and perimeter, length of the watershed, maximum and medium length of the watershed, relief ratio, form factor, medium slope of the watershed, mean slope width, length of the main stream in the watershed, total stream length, drainage density, total number of the length of a stream order, medium length of streams of same order, number of same stream order, stream frequency, bifurcation ratio, and drainage density for different stream orders of all 63 watersheds have been carried out using the standard mathematical formulae given in table 1 . The values of all morphometric parameters are shown in table 2 .

\section{Description of the project area}

The "Iron Gates" Natural Park is situated in the southwestern part of Romania and spans 115,655 ha along the Danube River in Southern Carpathians Mountains at the border with Serbia (Fig. 3). The counties of Caraș-Severin - western part, and Mehedinți - eastern part, almost equally share the park limits. The geographical location is between $21^{\circ} 21^{\prime}$ and $22^{\circ} 36^{\prime}$ ' eastern longitude and $44^{\circ} 51^{\prime}$ and $44^{\circ} 28^{\prime}$ northern latitude. 
"Iron Gates" Natural Park is an area of outstanding diversity of landscapes, which is the result of various interactions in time of natural elements (lithology, relief, climate, hydrography, vegetation and wildlife) and human activities. These interactions have contributed to shaping one of the most spectacular areas in Romania, from the scientific, cultural, recreational, and educational perspectives. Because of the human-nature relations that shaped a landscape of aesthetic, ecological and cultural values, the "Iron Gates" Natural Park has been declared as a protected area according to the IUCN category V.

The high diversity of species and habitats of community interests present here has brought to the designation of this area as Natura 2000 site, both proposed Special Areas of Conservation (for the protection of habitats and species according to EU Habitats Directives) and Special Protection Area (for the protection of birds according to EU Habitats Directives). According to the "Iron Gates" Natural Park Management Plan approved on the 11th December 2013, there are 18 protected areas of biological, landscape, and paleontological and geological importance. Out of these 18 areas, four are Special Protection Areas for birds and wetland conservation: Divici - Pojejena 498 ha (a succession of five ponds and swamp areas along the Danube River), Calinovăţ Island of 24 ha (located on the Danube River between Baziaş and Divici), Ostrov - Moldova Veche of 1,627 ha (a large island with many wetland areas) and Nera Marsh - Danube a mixt reserve of 10 ha (situated in the western extremity of the park at the confluence of Nera River with the Danube).

After the construction of the "Iron Gates" Dam, all the confluences of the Danube's tributary streams have been flooded and transformed in gulfs of different areas. The biggest gulfs are those of Cerna, Bahna, and Mraconia rivers. Upstream the effect of the dam was the flooding of the alluvial fans formed by the tributary rivers (Berzeasca, Cameniţa). As a result the water surface increased and new wetland and riparian habitats emerged, which are characterized by specific top climate with increased humidity, low temperatures, etc. Some of the new gulfs received a high input of sediments that contributed to the formation of microdeltas (Gornea - Sicheviţa, Cameniţa, Liuborajdea) with complex habitats necessary for the avian fauna and ichthyofauna.

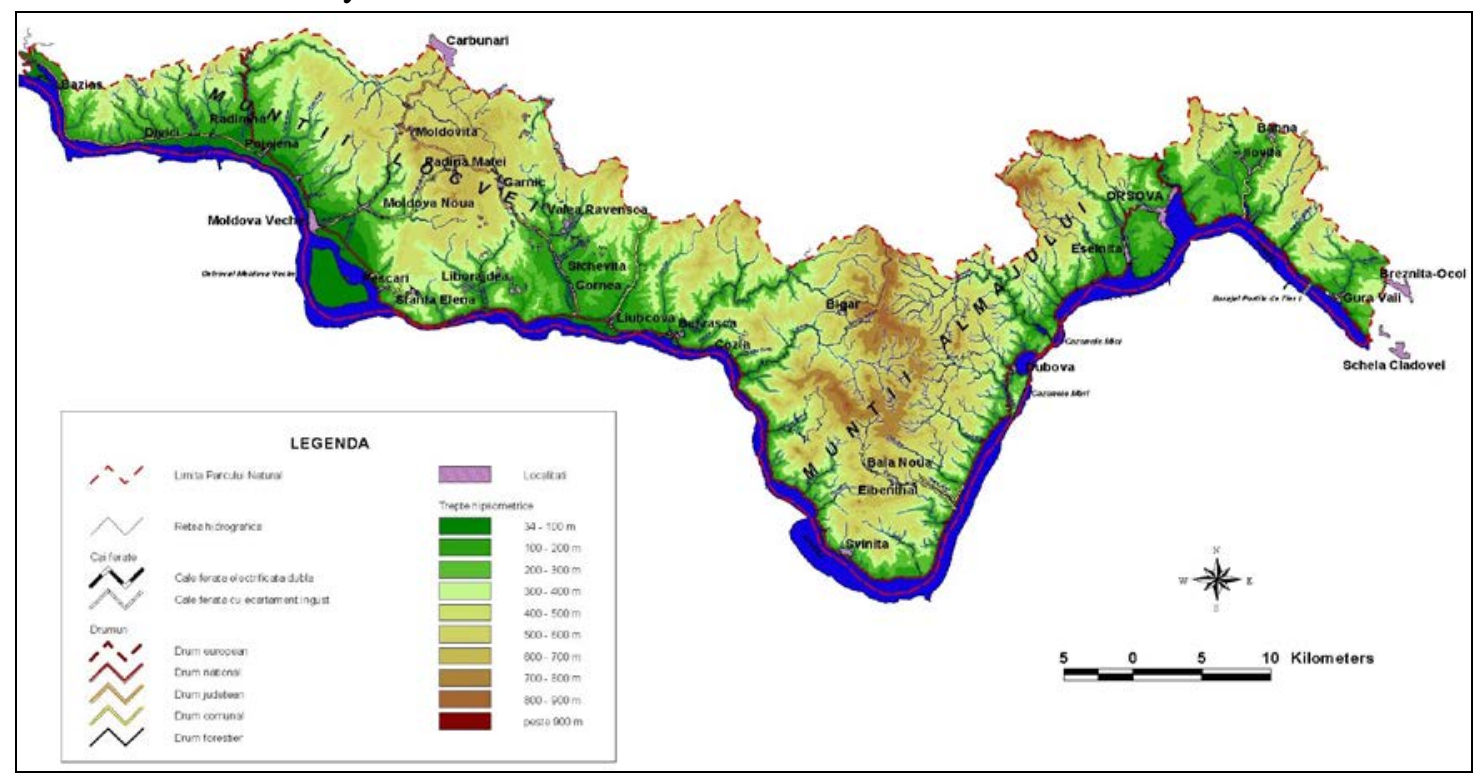

Figure 3: “Iron Gates” Natural Park - physical map. 
Table 1: Methodology used for analysis of the morphometric parameters.

\begin{tabular}{|c|c|c|c|}
\hline No. & Morphometric parameter & Formulae & Ref. \\
\hline 1. & Area of watershed (A) & Derived from the geometry of the delineated polygons in $\mathrm{km}^{2}$ & (18) \\
\hline 2. & Watershed perimeter (P) & Derived from the geometry of the delineated polygons in GIS & $(18)$ \\
\hline 3. & Watershed length (L) & $\begin{array}{l}\text { Distance from the pour point to a point at the watershed divide } \\
\text { on the direction of the main stream }\end{array}$ & (18) \\
\hline 4. & $\begin{array}{l}\text { Maximum watershed } \\
\text { width }\left(\mathrm{L}_{\max }\right)\end{array}$ & $\begin{array}{l}\text { The maximum length of a perpendicular line to the line of the } \\
\text { watershed length }\end{array}$ & $(18)$ \\
\hline 5. & $\begin{array}{l}\text { Mead width of watershed } \\
\text { (B) }\end{array}$ & $\begin{array}{l}\mathrm{B}=\mathrm{S} / \mathrm{L} \text {; Where, } \mathrm{B}=\text { mean width of basin; } \mathrm{A}=\text { basin area; } \mathrm{L}= \\
\text { basin length }\end{array}$ & $(16)$ \\
\hline 6. & Relief Ratio $\left(\mathrm{R}_{\mathrm{r}}\right)$ & $\begin{array}{l}\mathrm{R}_{\mathrm{r}}=\mathrm{h} / \mathrm{L} \text {; Where, } \mathrm{h}=\text { difference of altitude between the } \\
\text { highest point in the watershed and the pour point; } \mathrm{L}=\text { basin } \\
\text { length }\end{array}$ & $(17)$ \\
\hline 7. & Form factor $\left(\mathrm{R}_{\mathrm{f}}\right)$ & $\begin{array}{l}R_{f}=D_{c} / L \text {; Where, } D_{c}=\text { diameter of a circle with area equal to } \\
\text { watershed area }\left(D_{c}=\sqrt{ }(4 x S) / p\right) ; L=\text { watershed length }\end{array}$ & $(1)$ \\
\hline 8. & Circularity ratio $\left(\mathrm{R}_{\mathrm{c}}\right)$ & $\begin{array}{l}\mathrm{R}_{\mathrm{c}}=4^{*} \pi^{*} \mathrm{~A} / \mathrm{P}^{2} \text {; Where, } \mathrm{R}_{\mathrm{c}}=\text { circularity ratio; } \pi=\text { "Pi" value } \\
\text { that is } 3.14 ; \mathrm{A}=\text { basin area; } \mathrm{P}=\text { basin perimeter }\end{array}$ & $(10)$ \\
\hline 9. & $\begin{array}{l}\text { Mean slope of the } \\
\text { watershed }\left(\mathrm{S}_{\mathrm{b}}\right)\end{array}$ & $\begin{array}{l}\mathrm{S}_{\mathrm{b}}=(\text { alt. at } 0.85 \mathrm{~L}-\text { alt. at } 0.10 \mathrm{~L}) / 0.75 \mathrm{~L}\left(\mathrm{~S}_{\mathrm{b}} \text { in } \% \text { or units }\right) ; \mathrm{L}= \\
\text { watershed length }\end{array}$ & $(23)$ \\
\hline 10. & Mean slope width $\left(l_{v}\right)$ & $\mathrm{l}_{\mathrm{v}}=0.55 \times \mathrm{S} / \sum \mathrm{L}$; Where, $\mathrm{A}=$ watershed area; $\mathrm{L}=$ basin length & $(25)$ \\
\hline 11. & $\begin{array}{l}\text { Length of main stream } \\
\left(\mathrm{L}_{\text {râupp }}\right)\end{array}$ & Derived in ArcGIS from topographical maps & (19) \\
\hline 12. & $\begin{array}{l}\text { Total streams length } \\
\qquad\left(\sum \mathrm{L}\right)\end{array}$ & Calculated as the sum of the length of all rivers in a basin & (19) \\
\hline 13. & Drainage density $\left(\mathrm{D}_{\mathrm{d}}\right)$ & $\mathrm{D}_{\mathrm{d}}=\sum \mathrm{L} / \mathrm{S} ;$ Where, $\mathrm{L}=$ watershed length; $\mathrm{A}=$ basin area & $(16)$ \\
\hline 14. & $\begin{array}{l}\text { Number of streams of the } \\
\text { same order }\left(\mathrm{N}_{\mathrm{n}}\right)\end{array}$ & Where $\mathrm{n}=$ stream order & $(17)$ \\
\hline 15. & $\begin{array}{l}\text { Mean length of streams } \\
\text { of same order }\left(\mathrm{L}_{\text {medNn }}\right)\end{array}$ & Where $\mathrm{n}=$ stream order & $\begin{array}{l}(18, \\
19)\end{array}$ \\
\hline 16. & $\begin{array}{l}\text { Length of the streams of } \\
\text { same order }\left(\sum N_{n}\right)\end{array}$ & Where $\mathrm{n}=$ stream order & $(17)$ \\
\hline 17. & Stream frequency $\left(\mathrm{fN}_{\mathrm{n}}\right)$ & $\begin{array}{l}\mathrm{f}=\mathrm{N}_{\mathrm{n}} / \mathrm{S} \text {; Where, } \mathrm{N}=\text { number of stream segments of same } \\
\text { order; } \mathrm{A}=\text { watershed area }\end{array}$ & $(18)$ \\
\hline 18. & $\begin{array}{l}\text { Bifurcation Ratio }\left(\mathrm{R}_{\mathrm{b}}\right) \\
\text { Mean bifurc. ratio }\left(\mathrm{R}_{\mathrm{bm}}\right)\end{array}$ & $\begin{array}{l}\mathrm{R}_{\mathrm{b}}=\mathrm{N}_{\mathrm{u}} / \mathrm{N}_{\mathrm{u}+1} ; \text { Where, } \mathrm{N}_{1}=\text { total no. of stream segments of } \\
\text { order , } \mathrm{u} \text { ”; } \mathrm{N}_{\mathrm{u}+1}=\text { number of segments of the next higher order }\end{array}$ & (7) \\
\hline 19. & Drainage density $\left(D_{n}\right)$ & $\begin{array}{l}D_{n}=\sum L_{n} / S ; \text { Where, } n=\text { stream order; } L_{n}=\text { total length of } \\
\text { stream segments of same order; } S=\text { watershed area }\end{array}$ & $\begin{array}{l}(18, \\
19)\end{array}$ \\
\hline
\end{tabular}


Table 2: Morphometric characteristics of the river network in "Iron Gates" Natural Park (IGNP).

\begin{tabular}{|c|c|c|c|c|c|c|c|c|c|c|c|c|c|}
\hline \multicolumn{14}{|c|}{ Mountain range } \\
\hline \multicolumn{2}{|c|}{ Watershead } & 节 & 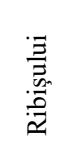 & 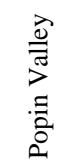 & 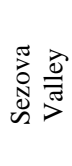 & 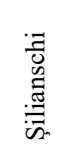 & 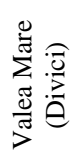 & 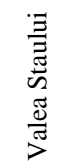 & 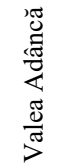 & 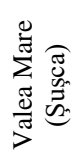 & 苂 & 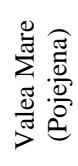 & 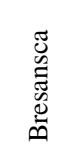 \\
\hline \multicolumn{2}{|c|}{$\begin{array}{c}\text { Danube } \\
\text { Confluence }\end{array}$} & $\mathrm{km}$ & $\begin{array}{c}\mathrm{km} \\
1068\end{array}$ & $\begin{array}{c}\mathrm{km} \\
1064\end{array}$ & $\begin{array}{c}\mathrm{km} \\
1063\end{array}$ & $\begin{array}{c}\mathrm{km} \\
1063\end{array}$ & $\begin{array}{c}\mathrm{km} \\
1061\end{array}$ & $\begin{array}{c}\mathrm{km} \\
1060\end{array}$ & $\begin{array}{c}\mathrm{km} \\
1059\end{array}$ & $\begin{array}{c}\mathrm{km} \\
1056\end{array}$ & $\begin{array}{c}\mathrm{km} \\
1055\end{array}$ & $\begin{array}{c}\mathrm{km} \\
1053\end{array}$ & $\begin{array}{c}\mathrm{km} \\
1051\end{array}$ \\
\hline \multirow{12}{*}{ 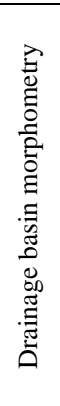 } & $\mathrm{A}$ & $\mathrm{km}^{2}$ & 6.56 & 1.3 & 0.98 & 2.01 & 5.8 & 6.6 & 4.24 & 9.55 & 81.6 & 25.0 & 2.5 \\
\hline & $\mathrm{P}$ & $\mathrm{km}$ & 11.8 & 5.6 & 0.49 & 7.5 & 14.9 & 14.1 & 12.4 & 16.2 & 70.8 & 24.7 & 8.3 \\
\hline & $\mathrm{L}$ & $\mathrm{km}$ & 4.17 & 2.27 & 2.1 & 3.16 & 5.32 & 5.84 & 5.32 & 6.44 & 25.7 & 7.61 & 3.66 \\
\hline & Lmax & $\mathrm{km}$ & 2.57 & 0.94 & 0.64 & 0.86 & 2.05 & 1.9 & 1.22 & 3.09 & 7.78 & 5.43 & 1.32 \\
\hline & $\mathrm{B}$ & $\mathrm{km}$ & 1.57 & 0.57 & 0.47 & 0.64 & 1.09 & 1.13 & 0.8 & 1.48 & 3.13 & 3.29 & 0.68 \\
\hline & $\mathrm{R}_{\mathrm{r}}$ & - & 0.1 & 0.17 & 0.14 & 0.12 & 0.08 & 0.08 & 0.08 & 0.07 & 0.03 & 0.07 & 0.09 \\
\hline & $\mathrm{R}_{\mathrm{f}}$ & - & 0.39 & 0.32 & 0.3 & 0.29 & 0.29 & 0.28 & 0.25 & 0.31 & 0.22 & 0.42 & 0.28 \\
\hline & $\mathrm{R}_{\mathrm{c}}$ & - & 0.59 & 0.52 & 51.3 & 0.45 & 0.33 & 0.42 & 0.35 & 0.46 & 0.20 & 0.52 & 0.46 \\
\hline & $\mathrm{S}_{\mathrm{b}}$ & $\%$ & 5.08 & 15.7 & 13.5 & 9.8 & 7.02 & 6 & 7.22 & 6.83 & 2.13 & 5.5 & 8.96 \\
\hline & $l_{\mathrm{v}}$ & $\mathrm{m}$ & 319 & 232 & 195 & 235 & 255 & 212 & 231 & 249 & 348 & 332 & 326 \\
\hline & Hmax & $\mathrm{m}$ & 506 & 457 & 359 & 459 & 507 & 510 & 510 & 512 & 722 & 592 & 407 \\
\hline & Hmin & $\mathrm{m}$ & 70 & 74 & 68 & 66 & 67 & 67 & 67 & 67 & 59 & 67 & 67 \\
\hline \multirow{33}{*}{ 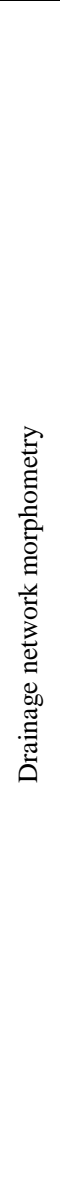 } & Lrâupp & $\mathrm{km}$ & 4.52 & 1.78 & 2.01 & 2.9 & 5.81 & 6.37 & 5.28 & 6.94 & 32.5 & 8.22 & 3.31 \\
\hline & $\sum \mathrm{L}$ & $\mathrm{km}$ & 11.3 & 3.08 & 2.76 & 4.71 & 12.5 & 17.1 & 10.1 & 21.1 & 129 & 41.4 & 4.22 \\
\hline & $\mathrm{D}_{\mathrm{d}}$ & $\mathrm{km} / \mathrm{km}^{2}$ & 1.73 & 2.37 & 2.82 & 2.34 & 2.16 & 2.6 & 2.38 & 2.21 & 1.54 & 1.66 & 1.69 \\
\hline & N1 & - & 16 & 4 & 2 & 4 & 15 & 18 & 11 & 17 & 112 & 37 & 2 \\
\hline & N2 & - & 5 & 1 & 1 & 1 & 3 & 5 & 3 & 6 & 32 & 11 & 1 \\
\hline & N3 & - & 1 & - & - & - & 1 & 1 & 1 & 2 & 6 & 5 & - \\
\hline & $\mathrm{N} 4$ & - & - & - & - & - & - & - & - & 1 & 2 & 1 & - \\
\hline & N5 & - & - & - & - & - & - & - & - & - & - & - & - \\
\hline & LmedN1 & $\mathrm{m}$ & 385 & 446 & 976 & 362 & 385 & 557 & 353 & 466 & 561 & 517 & 1,407 \\
\hline & LmedN2 & $\mathrm{m}$ & $\begin{array}{c}459 . \\
81\end{array}$ & $\begin{array}{c}1782 \\
.97\end{array}$ & $\begin{array}{c}810 . \\
99\end{array}$ & $\begin{array}{c}2905 \\
.74\end{array}$ & $\begin{array}{c}527 . \\
59\end{array}$ & $\begin{array}{c}407 . \\
06\end{array}$ & $\begin{array}{c}590 . \\
22\end{array}$ & $\begin{array}{c}1058 \\
.7\end{array}$ & $\begin{array}{c}1008 \\
.34\end{array}$ & $\begin{array}{c}706 . \\
71\end{array}$ & $\begin{array}{c}1410 \\
.63\end{array}$ \\
\hline & LmedN3 & $\mathrm{m}$ & $\begin{array}{c}2876 \\
.07\end{array}$ & - & - & - & $\begin{array}{c}4937 \\
.46\end{array}$ & $\begin{array}{c}5075 \\
.93\end{array}$ & $\begin{array}{c}4441 \\
.78\end{array}$ & $\begin{array}{c}2417 \\
.2\end{array}$ & $\begin{array}{c}3593 \\
.41\end{array}$ & 1868 & - \\
\hline & LmedN4 & $\mathrm{m}$ & - & - & - & - & - & - & - & $\begin{array}{c}1958 \\
.23 \\
\end{array}$ & $\begin{array}{c}2368 \\
.7 \\
\end{array}$ & $\begin{array}{c}5191 \\
.4 \\
\end{array}$ & - \\
\hline & LmedN5 & $\mathrm{m}$ & - & - & - & - & - & - & - & - & - & - & - \\
\hline & $\sum \mathrm{N} 1$ & $\mathrm{~km}$ & 6.15 & 1.78 & 1.95 & 1.80 & 5.8 & 10.0 & 3.87 & 7.92 & 58.3 & 19.1 & 2.81 \\
\hline & $\sum \mathrm{N} 2$ & $\mathrm{~km}$ & 2.29 & 1.72 & 0.82 & 2.9 & 1.6 & 2.03 & 1.77 & 6.35 & 26.3 & 7.73 & 1.41 \\
\hline & $\sum \mathrm{N} 3$ & $\mathrm{~km}$ & 2.87 & - & - & - & 4.9 & 5.07 & 4.44 & 4.83 & 21.0 & 9.34 & - \\
\hline & $\sum \mathrm{N} 4$ & $\mathrm{~km}$ & - & - & - & - & - & - & - & 1.96 & 23.5 & 5.2 & - \\
\hline & $\sum \mathrm{N} 5$ & $\mathrm{~km}$ & - & - & - & - & - & - & - & - & - & - & - \\
\hline & fN1 & - & 2.44 & 3.07 & 2.04 & 1.99 & 2.59 & 2.73 & 2.59 & 1.78 & 1.37 & 1.48 & 0.8 \\
\hline & fN2 & - & 0.76 & 0.76 & 1.02 & 0.5 & 0.52 & 0.76 & 0.71 & 0.63 & 0.39 & 0.44 & 0.4 \\
\hline & fN3 & - & 0.15 & - & - & - & 0.85 & 0.15 & 0.24 & 0.21 & 0.07 & 0.20 & - \\
\hline & fN4 & - & - & - & - & - & - & - & - & 0.1 & 0.02 & 0.04 & - \\
\hline & fN5 & - & - & - & - & - & - & - & - & - & - & - & - \\
\hline & $\mathrm{R}_{\mathrm{bN} 1}$ & - & 3.2 & 4 & 2 & 4 & 5 & 3.6 & 3.67 & 2.83 & 3.50 & 3.36 & 2 \\
\hline & $\mathrm{R}_{\mathrm{bN} 2}$ & - & 5 & - & - & - & 3 & 5 & 3 & 3 & 5.33 & 2.20 & - \\
\hline & $\mathrm{R}_{\mathrm{bN} 3}$ & - & - & - & - & - & - & - & - & 2 & 3.00 & 5.00 & - \\
\hline & $\mathrm{R}_{\mathrm{bN} 4}$ & - & - & - & - & - & - & - & $\begin{array}{lll}- & \\
-\end{array}$ & - & - & $\begin{array}{lll}- & \\
\end{array}$ & - \\
\hline & $\mathrm{R}_{\mathrm{bm}}$ & - & 2.1 & 1.0 & 0.5 & 1.0 & 2.0 & 2.2 & 1.7 & 2.0 & 3.0 & 2.6 & 0.5 \\
\hline & DdN1 & - & 0.94 & 1.37 & 1.99 & 0.9 & 0.99 & 1.52 & 0.91 & 0.83 & 0.71 & 0.76 & 1.12 \\
\hline & DdN2 & - & 0.35 & 1.32 & 0.84 & 1.44 & 0.27 & 0.31 & 0.41 & 0.66 & 0.32 & 0.31 & 0.56 \\
\hline & DdN3 & - & 0.44 & - & - & - & 0.85 & 0.77 & 1.05 & 0.51 & 0.26 & 0.37 & - \\
\hline & DdN4 & - & - & - & - & - & - & - & - & 0.21 & 0.29 & 0.21 & - \\
\hline & DdN5 & - & - & - & - & - & - & - & - & - & - & - & - \\
\hline
\end{tabular}


Table 2 (continued): Morphometric characteristics of the river network in the IGNP.

\begin{tabular}{|c|c|c|c|c|c|c|c|c|c|c|c|c|c|}
\hline \multicolumn{14}{|c|}{ Mountain range } \\
\hline \multicolumn{2}{|c|}{ Watershead } & 壱 & 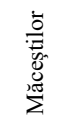 & $\sum_{\substack{n \\
\tilde{a}}}^{\pi}$ & 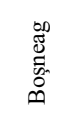 & 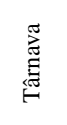 & 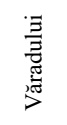 & 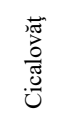 & $\stackrel{80}{\stackrel{0}{7}}$ & 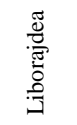 & 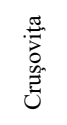 &  & 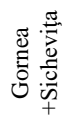 \\
\hline \multicolumn{2}{|c|}{ Danube Confluence } & $\mathrm{km}$ & $\begin{array}{c}\mathrm{km} \\
1050\end{array}$ & $\begin{array}{c}\mathrm{km} \\
1049\end{array}$ & $\begin{array}{c}\mathrm{km} \\
1045\end{array}$ & $\begin{array}{c}\mathrm{km} \\
1043\end{array}$ & $\begin{array}{c}\mathrm{km} \\
1042\end{array}$ & $\begin{array}{c}\mathrm{km} \\
1039\end{array}$ & $\begin{array}{c}\mathrm{km} \\
1034\end{array}$ & $\begin{array}{c}\mathrm{km} \\
1032\end{array}$ & $\begin{array}{c}\mathrm{km} \\
1030\end{array}$ & $\begin{array}{c}\mathrm{km} \\
1025\end{array}$ & $\begin{array}{c}\mathrm{km} \\
1024\end{array}$ \\
\hline \multirow{12}{*}{  } & $\mathrm{A}$ & $\mathrm{km}^{2}$ & 5.8 & 10.4 & 55.6 & 4.2 & 2.9 & 5.48 & 7.78 & 38.8 & 9.6 & 2.12 & 83.8 \\
\hline & $\mathrm{P}$ & $\mathrm{km}$ & 14.5 & 17.8 & 38.0 & 10.6 & 8.3 & 12.4 & 13.1 & 33.1 & 13.8 & 6.75 & 50.2 \\
\hline & $\mathrm{L}$ & $\mathrm{km}$ & 6.19 & 7.02 & 10.8 & 4.19 & 3.36 & 4.46 & 4.4 & 9.13 & 5.07 & 2.4 & 16 \\
\hline & Lmax & $\mathrm{km}$ & 1.46 & 2.1 & 8.71 & 1.52 & 1.36 & 1.8 & 2.59 & 8.27 & 3.05 & 1.69 & 28.4 \\
\hline & $\mathrm{B}$ & $\mathrm{km}$ & 0.94 & 1.48 & 5.13 & 1 & 0.86 & 1.23 & 1.77 & 4.25 & 1.89 & 0.88 & 5.24 \\
\hline & $\mathrm{R}_{\mathrm{r}}$ & - & 0.09 & 0.08 & 0.06 & 0.11 & 0.14 & 0.1 & 0.09 & 0.07 & 0.08 & 0.11 & 0.04 \\
\hline & $\mathrm{R}_{\mathrm{f}}$ & - & 0.25 & 0.29 & 0.44 & 0.31 & 0.32 & 0.33 & 0.4 & 0.43 & 0.39 & 0.39 & 0.36 \\
\hline & $\mathrm{R}_{\mathrm{c}}$ & - & 0.34 & 0.41 & 0.48 & 0.47 & 0.53 & 0.45 & 0.57 & 0.45 & 0.63 & 0.58 & 0.42 \\
\hline & $S_{b}$ & $\%$ & 8.4 & 6.7 & 5.8 & 10.0 & 17.3 & 9.18 & 8.24 & 7.08 & 4.1 & 7.11 & 3.6 \\
\hline & $l_{\mathrm{v}}$ & $\mathrm{m}$ & 299 & 376 & 416 & 416 & 469 & 441 & 323 & 375 & 244 & 186 & 371 \\
\hline & Hmax & $\mathrm{m}$ & 599 & 631 & 737 & 542 & 527 & 491 & 445 & 734 & 481 & 332 & 765 \\
\hline & Hmin & $\mathrm{m}$ & 67 & 67 & 67 & 66 & 67 & 66 & 61 & 50 & 66 & 65 & 58 \\
\hline \multirow{33}{*}{ 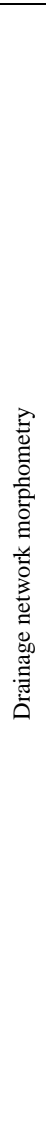 } & Lrâupp & $\mathrm{km}$ & 6.06 & 8.09 & 11.7 & 4.53 & 2.5 & 3.12 & 4.67 & 6.38 & 4 & 2.51 & 9.75 \\
\hline & $\sum \mathrm{L}$ & $\mathrm{km}$ & 10.7 & 15.2 & 73.5 & 5.55 & 3.4 & 6.83 & 13.3 & 56.9 & 21.7 & 6.26 & 124 \\
\hline & $\mathrm{D}_{\mathrm{d}}$ & $\mathrm{km} / \mathrm{km}^{2}$ & 1.84 & 1.46 & 1.32 & 1.32 & 1.17 & 1.25 & 1.7 & 1.47 & 2.26 & 2.95 & 1.48 \\
\hline & N1 & - & 12 & 7 & 58 & 2 & 2 & 7 & 21 & 54 & 25 & 11 & 127 \\
\hline & N2 & - & 3 & 2 & 21 & 1 & 1 & 1 & 5 & 17 & 14 & 2 & 40 \\
\hline & N3 & - & 1 & 1 & 2 & - & - & - & 2 & 7 & 2 & 1 & 8 \\
\hline & N4 & - & - & - & 1 & - & - & - & 1 & 2 & 1 & - & 2 \\
\hline & N5 & - & - & - & - & - & - & - & - & 1 & - & - & 1 \\
\hline & LmedN1 & $\mathrm{m}$ & $\begin{array}{c}334.8 \\
8\end{array}$ & $\begin{array}{c}937.1 \\
8\end{array}$ & $\begin{array}{c}645.7 \\
93\end{array}$ & $\begin{array}{c}1800 . \\
8\end{array}$ & $\begin{array}{l}768 \\
.84\end{array}$ & $\begin{array}{c}529 \\
.52\end{array}$ & $\begin{array}{c}309.7 \\
1\end{array}$ & $\begin{array}{c}137.3 \\
7\end{array}$ & $\begin{array}{c}435 \\
.92\end{array}$ & $\begin{array}{l}358 \\
.46\end{array}$ & $\begin{array}{c}455.4 \\
39\end{array}$ \\
\hline & LmedN2 & $\mathrm{m}$ & $\begin{array}{c}854 \\
.09\end{array}$ & $\begin{array}{c}1181 . \\
7\end{array}$ & $\begin{array}{c}1113 . \\
27\end{array}$ & $\begin{array}{c}1949 . \\
81\end{array}$ & $\begin{array}{c}1867 . \\
7\end{array}$ & $\begin{array}{c}3124 . \\
44\end{array}$ & 677.2 & $\begin{array}{c}833.5 \\
7\end{array}$ & $\begin{array}{c}707.4 \\
5\end{array}$ & $\begin{array}{c}770.0 \\
4\end{array}$ & $\begin{array}{c}806.0 \\
4\end{array}$ \\
\hline & LmedN3 & $\mathrm{m}$ & $\begin{array}{c}4100 . \\
69\end{array}$ & $\begin{array}{c}6291 . \\
1\end{array}$ & $\begin{array}{c}7432 . \\
7\end{array}$ & - & - & - & $\begin{array}{c}1201 . \\
3\end{array}$ & $\begin{array}{c}1227 . \\
9\end{array}$ & $\begin{array}{c}2311 . \\
8\end{array}$ & $\begin{array}{c}784.1 \\
9\end{array}$ & $\begin{array}{c}2128 . \\
07\end{array}$ \\
\hline & LmedN4 & $\mathrm{m}$ & - & - & $\begin{array}{c}4056 . \\
6\end{array}$ & - & - & - & $\begin{array}{c}967.0 \\
9\end{array}$ & $\begin{array}{c}2229 . \\
5\end{array}$ & $\begin{array}{c}1191 . \\
98\end{array}$ & - & $\begin{array}{c}3691 . \\
58\end{array}$ \\
\hline & LmedN5 & $\mathrm{m}$ & - & - & - & - & - & - & - & $\begin{array}{c}4458 . \\
9\end{array}$ & - & - & 9759 \\
\hline & $\sum \mathrm{N} 1$ & $\mathrm{~km}$ & 4.01 & 6.560 & 36.81 & 3.60 & 1.53 & 3.70 & 6.50 & 23.16 & 10.89 & 3.94 & 57.84 \\
\hline & $\sum \mathrm{N} 2$ & $\mathrm{~km}$ & 2.56 & 2.36 & 17.81 & 1.94 & 1.86 & 3.12 & 3.39 & 14.17 & 4.95 & 1.54 & 32.24 \\
\hline & $\sum \mathrm{N} 3$ & $\mathrm{~km}$ & 4.1 & 6.29 & 14.86 & - & - & - & 2.40 & 8.59 & 4.62 & 0.78 & 17.02 \\
\hline & $\sum \mathrm{N} 4$ & $\mathrm{~km}$ & - & - & 4.06 & - & - & - & 0.97 & 8.917 & 1.19 & - & 7.38 \\
\hline & $\sum \mathrm{N} 5$ & $\mathrm{~km}$ & - & - & - & - & - & - & - & 1.65 & - & - & 9.75 \\
\hline & fN1 & - & 2.05 & 0.67 & 1.04 & 0.48 & 0.69 & 1.28 & 2.7 & 1.39 & 2.6 & 5.19 & 1.51 \\
\hline & fN2 & - & 0.52 & 0.19 & 0.38 & 0.24 & 0.34 & 0.18 & 0.64 & 0.44 & 1.46 & 0.94 & 0.48 \\
\hline & fN3 & - & 0.17 & 0.1 & 0.04 & - & - & - & 0.26 & 0.18 & 0.21 & 0.47 & 0.1 \\
\hline & fN4 & - & - & - & 0.02 & - & - & - & 0.13 & 0.05 & 0.1 & - & 0.02 \\
\hline & fN5 & - & - & - & - & - & - & - & - & 0.03 & - & - & 0.01 \\
\hline & $\mathrm{R}_{\mathrm{bN1}}$ & - & 4 & 3.5 & 2.76 & 2 & 2 & 7 & 4.2 & 3.18 & 1.79 & 5.5 & 3.18 \\
\hline & $\mathrm{R}_{\mathrm{bN} 2}$ & - & 3 & 2 & 10.5 & - & - & - & 2.5 & 2.43 & 7 & 2 & 5 \\
\hline & $\mathrm{R}_{\mathrm{bN} 3}$ & - & - & - & 2 & - & - & - & 2 & 3.5 & 2 & - & 4 \\
\hline & $\mathrm{R}_{\mathrm{bN} 4}$ & - & - & - & - & - & - & - & - & 2 & - & - & 2 \\
\hline & $\mathrm{R}_{\mathrm{bm}}$ & - & 1.8 & 1.4 & 3.8 & 0.5 & 0.5 & 1.8 & 2.2 & 2.8 & 2.7 & 1.9 & 3.5 \\
\hline & DdN1 & - & 0.68 & 0.63 & 0.66 & 0.86 & 0.53 & 0.68 & 0.84 & 0.6 & 1.13 & 1.87 & 0.69 \\
\hline & DdN2 & - & 0.44 & 0.23 & 0.32 & 0.46 & 0.54 & 0.57 & 0.44 & 0.36 & 0.52 & 0.73 & 0.38 \\
\hline & DdN3 & - & 0.71 & 0.6 & 0.27 & - & - & - & 0.31 & 0.22 & 0.48 & 0.37 & 0.2 \\
\hline & DdN4 & - & - & - & 0.07 & - & - & - & 0.12 & 0.23 & 0.12 & - & 0.09 \\
\hline & DdN5 & - & - & - & - & - & - & - & - & 0.04 & - & - & 0.12 \\
\hline
\end{tabular}


Table 2 (continued): Morphometric characteristics of the river network in the IGNP.

\begin{tabular}{|c|c|c|c|c|c|c|c|c|c|c|c|c|c|c|}
\hline \multicolumn{15}{|c|}{ Mountain range } \\
\hline \multicolumn{2}{|c|}{ Watershead } & 营 & $\begin{array}{l}\stackrel{5}{5} \\
\frac{\pi}{0} \\
0 \\
0\end{array}$ & 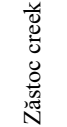 &  & 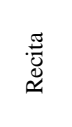 & $\frac{\pi}{\pi}$ & $\stackrel{\mathscr{\Xi}}{\stackrel{\Xi}{\Xi}}$ & 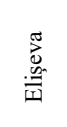 & 䒬 & 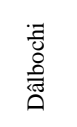 & 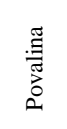 & 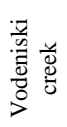 & 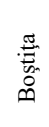 \\
\hline \multicolumn{2}{|c|}{$\begin{array}{c}\text { Danube } \\
\text { Confluence }\end{array}$} & $\mathrm{km}$ & $\begin{array}{c}\mathrm{km} \\
1023\end{array}$ & $\begin{array}{c}\mathrm{km} \\
1020\end{array}$ & $\begin{array}{c}\mathrm{km} \\
1018\end{array}$ & $\begin{array}{c}\mathrm{km} \\
1015\end{array}$ & $\begin{array}{c}\mathrm{km} \\
1013\end{array}$ & $\begin{array}{c}\mathrm{km} \\
1012\end{array}$ & $\begin{array}{c}\mathrm{km} \\
1007\end{array}$ & $\begin{array}{c}\mathrm{km} \\
1001\end{array}$ & $\begin{array}{c}\mathrm{km} \\
998.6\end{array}$ & $\begin{array}{l}\mathrm{km} \\
998\end{array}$ & $\begin{array}{l}\mathrm{km} \\
993\end{array}$ & $\begin{array}{l}\mathrm{km} \\
990\end{array}$ \\
\hline \multirow{12}{*}{ 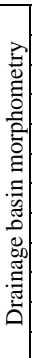 } & $\mathrm{A}$ & $\mathrm{km}^{2}$ & 102 & 1.51 & 229 & 6.6 & 3.04 & 74.2 & 20.22 & 14.23 & 1.1 & 6.6 & 1.71 & 1.7 \\
\hline & $\mathrm{P}$ & $\mathrm{km}$ & 28.1 & 6.6 & 245 & 13.76 & 7.59 & 46.8 & 20.19 & 18.71 & 4.4 & 11.7 & 6.29 & 6.1 \\
\hline & $\mathrm{L}$ & $\mathrm{km}$ & 20.3 & 2.42 & 26.28 & 5.62 & 3.08 & 13.4 & 6.081 & 6.46 & 1.39 & 4.03 & 2.43 & 2.5 \\
\hline & Lmax & $\mathrm{km}$ & 9.07 & 1.31 & 14.51 & 2.01 & 1.45 & 8.61 & 4.11 & 4.07 & 1.04 & 2.5 & 1.01 & 1.1 \\
\hline & $\mathrm{B}$ & $\mathrm{km}$ & 5.03 & 0.62 & 8.71 & 1.17 & 0.99 & 5.54 & 3.33 & 2.2 & 0.79 & 1.64 & 0.7 & 0.7 \\
\hline & $\mathrm{R}_{\mathrm{r}}$ & - & 0.04 & 0.13 & 0.04 & 0.11 & 0.2 & 0.06 & 0.14 & 0.11 & 0.23 & 0.17 & 0.24 & 0.2 \\
\hline & $\mathrm{R}_{\mathrm{f}}$ & - & 0.32 & 0.32 & 0.37 & 0.29 & 0.36 & 0.41 & 0.47 & 0.37 & 0.48 & 0.41 & 0.34 & 0.3 \\
\hline & $\mathrm{R}_{\mathrm{c}}$ & - & 1.62 & 0.44 & 0.05 & 0.44 & 0.66 & 0.43 & 0.62 & 0.51 & 0.71 & 0.61 & 0.54 & 0.6 \\
\hline & $\mathrm{S}_{\mathrm{b}}$ & $\%$ & 4.2 & 11.57 & 2.6 & 9.8 & 19.26 & 5.73 & 11.25 & 9.7 & 18.23 & 13.46 & 18.66 & 23 \\
\hline & $l_{\mathrm{v}}$ & $\mathrm{m}$ & 326 & 281 & 295 & 283 & 398 & 277 & 358 & 310 & 189 & 300 & 399 & 288 \\
\hline & Hmax & $\mathrm{m}$ & 852 & 373 & 1068 & 687 & 659 & 874.5 & 908 & 776 & 392 & 752 & 651 & 600 \\
\hline & Hmin & $\mathrm{m}$ & 66 & 66 & 54 & 65 & 51 & 48 & 61 & 61 & 68 & 63 & 67 & 70 \\
\hline \multirow{33}{*}{ 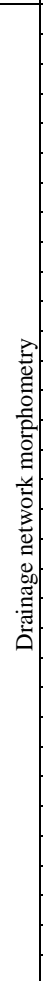 } & Lrâupp & $\mathrm{km}$ & 25 & 2.24 & 48.56 & 6.17 & 3.34 & 21.27 & 6.94 & 6.56 & 1.38 & 4.42 & 1.97 & 1.8 \\
\hline & $\sum \mathrm{L}$ & $\mathrm{km}$ & 172.3 & 2.96 & 427.2 & 12.83 & 4.2 & 147.5 & 31.09 & 25.28 & 3.2 & 12.08 & 2.36 & 3.3 \\
\hline & $\mathrm{D}_{\mathrm{d}}$ & $\mathrm{km} / \mathrm{km}^{2}$ & 1.69 & 1.96 & 1.87 & 1.94 & 1.38 & 1.99 & 1.54 & 1.78 & 2.91 & 1.83 & 1.38 & 1.9 \\
\hline & N1 & - & 159 & 3 & 503 & 11 & 2 & 167 & 34 & 30 & 6 & 14 & 2 & 6 \\
\hline & N2 & - & 51 & 1 & 129 & 3 & 1 & 46 & 9 & 7 & 2 & 6 & 1 & 1 \\
\hline & N3 & - & 13 & - & 26 & 1 & - & 9 & 2 & 1 & 1 & 1 & - & - \\
\hline & N4 & - & 3 & - & 5 & - & - & 3 & 1 & - & - & - & - & - \\
\hline & N5 & - & 1 & - & 1 & - & - & 1 & - & - & - & - & - & - \\
\hline & LmedN1 & $\mathrm{m}$ & 536.5 & 440.5 & 516.2 & 447.6 & 1230 & 468.1 & 508.6 & 526.6 & 385.7 & 452.5 & 353.2 & 261 \\
\hline & LmedN2 & $\mathrm{m}$ & 829.8 & 1646 & 683.7 & 1676 & 1740 & 679 & 869.2 & 584.7 & 123.5 & 454.7 & 1657 & 1782 \\
\hline & LmedN3 & $\mathrm{m}$ & 1474 & - & 2346 & 2877 & - & 1643 & 1797 & 5397 & 642.4 & 3024 & - & - \\
\hline & LmedN4 & $\mathrm{m}$ & 2705 & - & 5882 & - & - & 2082 & 2379 & - & - & - & - & - \\
\hline & LmedN5 & $\mathrm{m}$ & 17421 & - & 33893 & - & - & 17.265 & - & - & - & - & - & - \\
\hline & $\sum \mathrm{N} 1$ & $\mathrm{~km}$ & 85.30 & 1.32 & 259.7 & 4.920 & 2.46 & 78.17 & 17.29 & 15.79 & 2.31 & 6.33 & 0.71 & 1.6 \\
\hline & $\sum \mathrm{N} 2$ & $\mathrm{~km}$ & 42.31 & 1.64 & 88.20 & 5.03 & 1.74 & 31.23 & 7.82 & 4.092 & 0.246 & 2.72 & 1.65 & 1.8 \\
\hline & $\sum \mathrm{N} 3$ & $\mathrm{~km}$ & 19.16 & - & 61.00 & 2.87 & - & 14.78 & 3.59 & 5.39 & 0.64 & 3.02 & - & - \\
\hline & $\sum \mathrm{N} 4$ & $\mathrm{~km}$ & 8.115 & - & 29.41 & - & - & 62.46 & 2.37 & - & - & - & - & - \\
\hline & $\sum \mathrm{N} 5$ & $\mathrm{~km}$ & 17.42 & - & 33.89 & - & - & 17.26 & - & - & - & - & - & - \\
\hline & fN1 & - & 1.56 & 1.98 & 2.20 & 1.67 & 0.66 & 2.25 & 1.68 & 2.11 & 5.45 & 2.12 & 1.17 & 3.4 \\
\hline & fN2 & - & 0.5 & 0.66 & 0.56 & 0.45 & 0.33 & 0.62 & 0.45 & 0.49 & 1.82 & 2.72 & 0.58 & 0.6 \\
\hline & fN3 & - & 0.13 & - & 0.11 & 0.15 & - & 0.12 & 0.1 & 0.07 & 0.91 & 3.02 & - & - \\
\hline & fN4 & - & 0.03 & - & 0.02 & - & - & 0.04 & 0.05 & - & - & - & - & - \\
\hline & fN5 & - & 0.05 & - & 0.004 & - & - & 0.01 & - & - & - & - & - & - \\
\hline & $\mathrm{R}_{\mathrm{bN} 1}$ & - & 3.12 & 3 & 3.90 & 3.67 & 2 & 3.63 & 3.78 & 4.29 & 3 & 2.33 & 2 & 6 \\
\hline & $\mathrm{R}_{\mathrm{bN} 2}$ & - & 3.92 & - & 4.96 & 3 & - & 5.11 & 4.5 & 7 & 2 & 6 & - & - \\
\hline & $\mathrm{R}_{\mathrm{bN} 3}$ & - & 4.33 & - & 5.20 & - & - & 3 & 2 & - & - & - & - & - \\
\hline & $\mathrm{R}_{\mathrm{bN} 4}$ & - & 3 & - & 5.00 & - & - & 3 & - & - & - & - & - & - \\
\hline & $\mathrm{R}_{\mathrm{bm}}$ & - & 3.6 & 0.8 & 4.8 & 1.7 & 0.5 & 3.7 & 2.6 & 2.8 & 1.3 & 2.1 & 0.5 & 1.5 \\
\hline & DdN1 & - & 1.2 & 0.87 & 1.13 & 0.75 & 0.81 & 1.05 & 0.86 & 1.11 & 2.1 & 0.96 & 0.41 & 0.89 \\
\hline & DdN2 & - & 2.41 & 1.09 & 0.39 & 0.76 & 0.57 & 0.42 & 0.39 & 0.29 & 0.22 & 0.41 & 0.96 & 1.0 \\
\hline & DdN3 & - & 5.32 & - & 0.27 & 0.43 & - & 0.2 & 0.18 & 0.38 & 0.58 & 0.46 & - & - \\
\hline & DdN4 & - & 12.57 & - & 0.13 & - & - & 0.84 & 0.12 & - & - & - & - & - \\
\hline & DdN5 & - & 5.86 & - & 0.15 & - & - & 0.23 & - & - & - & - & - & - \\
\hline
\end{tabular}


Table 2 (continued): Morphometric characteristics of the river network in the IGNP.

\begin{tabular}{|c|c|c|c|c|c|c|c|c|c|c|c|c|c|}
\hline \multicolumn{14}{|c|}{ Mountain range } \\
\hline \multicolumn{2}{|c|}{ Watershead } & 总 & 寻 & 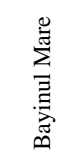 & 㺃 & 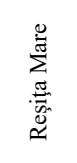 & $\begin{array}{l}\text { 芯 } \\
\text { 节 } \\
\end{array}$ & 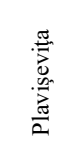 & 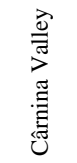 & 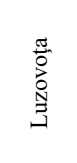 & 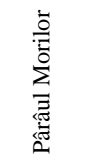 & 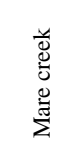 & 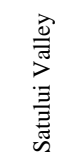 \\
\hline \multicolumn{2}{|c|}{$\begin{array}{c}\text { Danube } \\
\text { Confluence }\end{array}$} & $\mathrm{km}$ & $\begin{array}{l}\mathrm{km} \\
988 \\
\end{array}$ & $\begin{array}{l}\mathrm{km} \\
985\end{array}$ & $\begin{array}{l}\mathrm{km} \\
983\end{array}$ & $\begin{array}{c}\mathrm{km} \\
981.6\end{array}$ & $\begin{array}{l}\mathrm{km} \\
980\end{array}$ & $\begin{array}{l}\mathrm{km} \\
976\end{array}$ & $\begin{array}{c}\mathrm{km} \\
948.8\end{array}$ & $\begin{array}{l}\mathrm{km} \\
974\end{array}$ & $\begin{array}{c}\mathrm{km} \\
972.7\end{array}$ & $\begin{array}{c}\mathrm{km} \\
970.2\end{array}$ & $\begin{array}{l}\mathrm{km} \\
970\end{array}$ \\
\hline \multirow{12}{*}{ 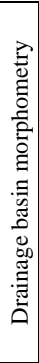 } & $\mathrm{A}$ & $\mathrm{km}^{2}$ & 12.22 & 1.49 & 30.47 & 3.08 & 14.1 & 18.33 & 3.8 & 1.95 & 19.48 & 1.1 & 8.97 \\
\hline & $\mathrm{P}$ & $\mathrm{km}$ & 18.93 & 5.4 & 31.93 & 9.4 & 18.63 & 21.31 & 10 & 5.8 & 22.45 & 5.1 & 14.6 \\
\hline & $\mathrm{L}$ & $\mathrm{km}$ & 7.16 & 2.13 & 11.22 & 4.09 & 7.06 & 7.02 & 4.28 & 2.39 & 8.49 & 1.3 & 4.85 \\
\hline & Lmax & $\mathrm{km}$ & 2.83 & 1.59 & 4.03 & 0.15 & 3.31 & 5.36 & 1.23 & 1.18 & 3.52 & 1.2 & 2.77 \\
\hline & B & $\mathrm{km}$ & 1.71 & 0.7 & 2.71 & 0.75 & 2 & 2.61 & 0.89 & 0.82 & 2.29 & 0.85 & 1.85 \\
\hline & $\mathrm{R}_{\mathrm{r}}$ & - & 0.09 & 0.22 & 0.07 & 0.12 & 0.1 & 0.11 & 0.14 & 0.18 & 0.09 & 0.24 & 0.15 \\
\hline & $\mathrm{R}_{\mathrm{f}}$ & - & 0.31 & 0.37 & 0.31 & 0.27 & 0.34 & 0.39 & 0.29 & 0.37 & 0.33 & 0.51 & 0.39 \\
\hline & $\mathrm{R}_{\mathrm{c}}$ & - & 0.43 & 0.64 & 0.38 & 0.44 & 0.51 & 0.51 & 0.48 & 0.73 & 0.49 & 0.53 & 0.53 \\
\hline & $\mathrm{S}_{\mathrm{b}}$ & $\%$ & 8.88 & 24.6 & 3.56 & 11.14 & 8.28 & 9.2 & 13.27 & 18.8 & 5.3 & 2.67 & 7.59 \\
\hline & $\mathrm{l}_{\mathrm{v}}$ & $\mathrm{m}$ & 313 & 264 & 310 & 239 & 280 & 205 & 269 & 228 & 220 & 240 & 213 \\
\hline & Hmax & $\mathrm{m}$ & 747 & 531 & 890 & 553 & 803 & 803 & 663 & 500 & 800 & 369 & 793 \\
\hline & Hmin & $\mathrm{m}$ & 77 & 59 & 62 & 62 & 62 & 60 & 60 & 61 & 76 & 60 & 59 \\
\hline \multirow{33}{*}{ 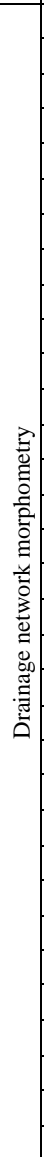 } & Lrâupp & $\mathrm{km}$ & 7.32 & 1.13 & 16 & 4.05 & 4.45 & 8.21 & 2.43 & 2.24 & 9.5 & 1.17 & 5.17 \\
\hline & $\sum \mathrm{L}$ & $\mathrm{km}$ & 21.5 & 3.1 & 54.11 & 7.1 & 27.7 & 49.2 & 7.77 & 4.7 & 48.67 & 2.52 & 23.2 \\
\hline & $\mathrm{D}_{\mathrm{d}}$ & $\mathrm{km} / \mathrm{km}^{2}$ & 1.76 & 2.08 & 1.78 & 2.31 & 1.96 & 2.68 & 2.05 & 2.41 & 2.5 & 2.29 & 2.59 \\
\hline & N1 & - & 23 & 3 & 63 & 10 & 29 & 71 & 6 & 3 & 58 & 4 & 29 \\
\hline & N2 & - & 13 & 1 & 15 & 4 & 7 & 20 & 2 & 1 & 30 & 1 & 6 \\
\hline & N3 & - & 1 & - & 2 & 1 & 1 & 4 & 1 & - & 12 & - & 2 \\
\hline & N4 & - & - & - & - & - & 1 & 1 & - & - & 1 & - & 1 \\
\hline & N5 & - & - & - & - & - & - & - & - & - & - & - & - \\
\hline & LmedN1 & $\mathrm{m}$ & 468.48 & 725.48 & 474.2 & 264.44 & 540.56 & 395.04 & 437.13 & 1219.2 & 423.36 & 338.5 & 451.52 \\
\hline & LmedN2 & $\mathrm{m}$ & 399.29 & 926.91 & 791.48 & 757.41 & 634.44 & 554.22 & 1064.3 & 1042.5 & 824.5 & 1174.1 & 924.6 \\
\hline & LmedN3 & $\mathrm{m}$ & 5538.4 & - & 15059 & 1435.2 & 4883.3 & 1184.1 & 1709.2 & - & 1386.9 & - & 1082.1 \\
\hline & LmedN4 & $\mathrm{m}$ & - & - & - & - & 2702.7 & 5337 & - & - & 7851.9 & - & 930.96 \\
\hline & LmedN5 & $\mathrm{m}$ & - & - & - & - & 15.67 & - & - & - & - & - & - \\
\hline & $\sum \mathrm{N} 1$ & $\mathrm{~km}$ & 10.77 & 2.17 & 27.98 & 2.64 & 4.44 & 28.05 & 3.93 & 3 & 24.55 & 1.35 & 1.31 \\
\hline & $\sum \mathrm{N} 2$ & $\mathrm{~km}$ & 5.19 & 0.92 & 11.08 & 3.029 & 4.88 & 11.084 & 2.12 & 1 & 10.71 & 1.17 & 5.54 \\
\hline & $\sum \mathrm{N} 3$ & $\mathrm{~km}$ & 5.538 & - & 15.058 & 1.4 & 2.702 & 4.73 & 1.7 & - & 5.54 & - & 2.16 \\
\hline & $\sum \mathrm{N} 4$ & $\mathrm{~km}$ & - & - & - & - & - & 5.337 & - & - & 7.85 & - & 0.93 \\
\hline & $\sum \mathrm{N} 5$ & $\mathrm{~km}$ & - & - & - & - & - & - & - & - & - & - & - \\
\hline & fN1 & - & 1.88 & 2.01 & 2.07 & 3.25 & 2.06 & 3.87 & 1.58 & 1.54 & 2.98 & 3.64 & 3.23 \\
\hline & fN2 & - & 1.06 & 0.67 & 0.49 & 1.3 & 0.5 & 1.09 & 0.53 & 1.04 & 1.54 & 0.91 & 0.67 \\
\hline & fN3 & - & 0.08 & - & 0.07 & 0.32 & 0.07 & 0.22 & 0.26 & - & 0.62 & - & 0.22 \\
\hline & fN4 & - & - & - & - & - & 0.07 & 0.05 & - & - & 0.05 & - & 0.11 \\
\hline & fN5 & - & - & - & - & - & - & - & - & - & - & - & - \\
\hline & $\mathrm{R}_{\mathrm{bN1}}$ & - & 1.77 & 3 & 4.2 & 2.5 & 4.14 & 3.55 & 3 & 3 & 1.93 & 4 & 4.83 \\
\hline & $\mathrm{R}_{\mathrm{bN} 2}$ & - & 13 & - & 7.5 & 4 & 7 & 5 & 2 & - & 2.5 & - & 3 \\
\hline & $\mathrm{R}_{\mathrm{bN} 3}$ & - & - & - & - & - & 1 & 4 & - & - & 12 & - & 2 \\
\hline & $\mathrm{R}_{\mathrm{bN} 4}$ & - & - & - & - & - & - & - & - & - & - & - & - \\
\hline & $\mathrm{R}_{\mathrm{bm}}$ & - & 3.7 & 0.8 & 2.9 & 1.6 & 3.0 & 3.1 & 1.3 & 0.8 & 4.1 & 1.0 & 2.5 \\
\hline & DdN1 & - & 0.88 & 1.46 & 0.92 & 0.86 & 1.11 & 1.53 & 1.03 & 1.87 & 1.26 & 1.23 & 0.15 \\
\hline & DdN2 & - & 0.42 & 0.62 & 0.36 & 0.98 & 0.31 & 0.6 & 0.56 & 0.53 & 0.55 & 1.06 & 0.62 \\
\hline & DdN3 & - & 0.45 & - & 0.49 & 0.45 & 0.35 & 0.26 & 0.45 & - & 0.28 & - & 0.24 \\
\hline & DdN4 & - & - & - & - & - & 0.19 & 0.29 & - & - & 0.4 & - & 0.1 \\
\hline & DdN5 & - & - & - & - & - & - & - & - & - & - & - & - \\
\hline
\end{tabular}


Table 2 (continued): Morphometric characteristics of the river network in the IGNP.

\begin{tabular}{|c|c|c|c|c|c|c|c|c|c|c|c|c|}
\hline \multicolumn{13}{|c|}{ Mountain range } \\
\hline \multicolumn{2}{|c|}{ Watershed } & 壱 & 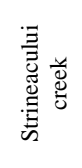 & 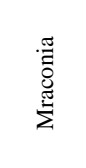 & 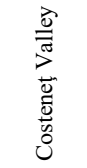 & 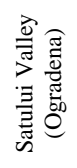 & $\begin{array}{l}\text { 훙 } \\
\text { 응 } \\
\text { in }\end{array}$ & $\sum^{\frac{\pi}{\pi}}$ & 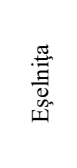 & 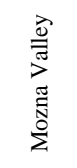 & 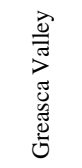 & 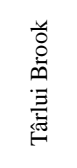 \\
\hline \multicolumn{2}{|c|}{$\begin{array}{c}\text { Danube } \\
\text { Confluence }\end{array}$} & $\mathrm{km}$ & $\begin{array}{c}\mathrm{km} \\
969.5\end{array}$ & $\begin{array}{l}\mathrm{km} \\
967\end{array}$ & $\begin{array}{c}\mathrm{km} \\
964.4\end{array}$ & $\begin{array}{c}\mathrm{km} \\
963.6\end{array}$ & $\begin{array}{c}\mathrm{km} \\
962.7\end{array}$ & $\begin{array}{l}\mathrm{km} \\
961\end{array}$ & $\begin{array}{l}\mathrm{km} \\
960\end{array}$ & $\begin{array}{c}\mathrm{km} \\
956.4\end{array}$ & $\begin{array}{c}\mathrm{km} \\
965.5\end{array}$ & $\begin{array}{l}\mathrm{km} \\
955\end{array}$ \\
\hline \multirow{12}{*}{ 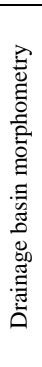 } & $\mathrm{A}$ & $\mathrm{km}^{2}$ & 2.8 & 113 & 3.8 & 9.69 & 5.7 & 17.74 & 48.61 & 0.8 & 2.3 & 4.6 \\
\hline & $\mathrm{P}$ & $\mathrm{km}$ & 7.6 & 42.6 & 10.1 & 15.76 & 11.26 & 24.46 & 39.35 & 3.8 & 6.6 & 11.67 \\
\hline & $\mathrm{L}$ & $\mathrm{km}$ & 2.4 & 13.42 & 4.19 & 5.71 & 4.4 & 9.13 & 20.66 & 1.34 & 2.3 & 4.25 \\
\hline & Lmax & $\mathrm{km}$ & 1.6 & 12.24 & 1.29 & 2.94 & 1.7 & 2.84 & 5.74 & 0.8 & 1.54 & 2.06 \\
\hline & $\mathrm{B}$ & $\mathrm{km}$ & 1.17 & 8.42 & 0.91 & 1.7 & 0.13 & 1.94 & 2.35 & 0.6 & 1 & 1.08 \\
\hline & $\mathrm{R}_{\mathrm{r}}$ & - & 0.16 & 0.06 & 0.1 & 0.1 & 0.11 & 0.09 & 0.04 & 0.12 & 0.1 & 0.1 \\
\hline & $\mathrm{R}_{\mathrm{f}}$ & - & 0.44 & 0.5 & 0.3 & 0.35 & 0.35 & 0.29 & 0.21 & 0.43 & 0.42 & 0.32 \\
\hline & $\mathrm{R}_{\mathrm{c}}$ & - & 0.61 & 0.78 & 0.47 & 0.49 & 0.56 & 0.37 & 0.39 & 0.70 & 0.66 & 0.42 \\
\hline & $S_{b}$ & $\%$ & 18.67 & 5.58 & 9.67 & 9.9 & 10.85 & 7.16 & 5.12 & 10.2 & 7.4 & 7.97 \\
\hline & $l_{\mathrm{v}}$ & $\mathrm{m}$ & 157 & 208 & 162 & 220 & 189 & 179 & 166 & 171 & 225 & 218 \\
\hline & Hmax & $\mathrm{m}$ & 455 & 820 & 483 & 643 & 563 & 905 & 969 & 230 & 291 & 489 \\
\hline & Hmin & $\mathrm{m}$ & 62 & 69 & 59 & 60 & 60 & 59 & 59 & 68 & 65 & 62 \\
\hline \multirow{33}{*}{ 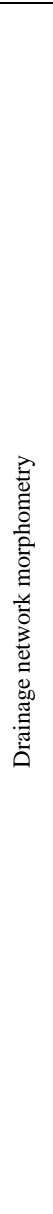 } & Lrâupp & $\mathrm{km}$ & 1.16 & 19 & 4.47 & 6.38 & 4.41 & 8.8 & 24.47 & 1.007 & 2.1 & 4.33 \\
\hline & $\sum \mathrm{L}$ & $\mathrm{km}$ & 9.84 & 299.18 & 12.94 & 24.24 & 16.56 & 54.45 & 160.78 & 2.58 & 5.62 & 11.61 \\
\hline & $\mathrm{D}_{\mathrm{d}}$ & $\mathrm{km} / \mathrm{km}^{2}$ & 3.51 & 2.65 & 3.41 & 2.5 & 2.91 & 3.07 & 3.31 & 3.23 & 2.44 & 2.52 \\
\hline & N1 & - & 15 & 399 & 15 & 31 & 20 & 82 & 178 & 4 & 7 & 16 \\
\hline & N2 & - & 5 & 108 & 7 & 7 & 4 & 20 & 53 & 2 & 3 & 3 \\
\hline & N3 & - & 1 & 25 & 1 & 2 & 1 & 4 & 17 & 1 & 1 & 1 \\
\hline & N4 & - & - & 6 & - & 1 & - & 1 & 2 & - & - & - \\
\hline & N5 & - & - & 1 & - & - & - & - & 1 & - & - & - \\
\hline & LmedN1 & $\mathrm{m}$ & 375.22 & 407.7 & 482.83 & 443.59 & 530.03 & 353.38 & 480.54 & 189.88 & 471.48 & 364.35 \\
\hline & LmedN2 & $\mathrm{m}$ & 662.08 & 605.44 & 458.38 & 429.35 & 842.45 & 732.56 & 663.06 & 469.39 & 268.95 & 1094.2 \\
\hline & LmedN3 & $\mathrm{m}$ & 468.72 & 1275.4 & 2492.9 & 2893.8 & 2595.7 & 491.62 & 1032.6 & 359.88 & 1515.4 & 1501.8 \\
\hline & LmedN4 & $\mathrm{m}$ & - & 4317.3 & - & 1696.3 & - & 6671.7 & 1233.4 & - & - & - \\
\hline & LmedN5 & $\mathrm{m}$ & - & 13333 & - & - & - & - & 10.043 & - & - & - \\
\hline & $\sum \mathrm{N} 1$ & $\mathrm{~km}$ & 5.62 & 162.67 & 7.24 & 13.75 & 10.60 & 28.97 & 85.54 & 0.75 & 3.30 & 5.82 \\
\hline & $\sum \mathrm{N} 2$ & $\mathrm{~km}$ & 3.31 & 65.39 & 3.2 & 30.05 & 3.36 & 14.65 & 35.14 & 0.93 & 0.806 & 3.28 \\
\hline & $\sum \mathrm{N} 3$ & $\mathrm{~km}$ & 0.46 & 31.88 & 2.29 & 5.78 & 2.59 & 1.97 & 17.55 & 0.36 & 1.51 & 1.501 \\
\hline & $\sum \mathrm{N} 4$ & $\mathrm{~km}$ & - & 25.90 & - & 1.696 & - & 6.67 & 2.47 & - & - & - \\
\hline & $\sum \mathrm{N} 5$ & $\mathrm{~km}$ & - & 13.33 & - & - & - & - & 20.09 & - & - & - \\
\hline & fN1 & - & 5 & 3.53 & 3.95 & 3.2 & 3.51 & 4.62 & 3.36 & 5 & 3.04 & 3.48 \\
\hline & fN2 & - & 1.07 & 0.96 & 1.84 & 0.72 & 0.7 & 1.13 & 1.09 & 2.5 & 1.3 & 0.65 \\
\hline & fN3 & - & 1.36 & 0.22 & 0.26 & 0.21 & 0.18 & 0.23 & 0.35 & 1.25 & 0.43 & 0.22 \\
\hline & fN4 & - & - & 0.05 & - & 0.1 & - & 0.06 & 0.04 & - & - & - \\
\hline & fN5 & - & - & 0.01 & - & - & - & - & 0.02 & - & - & - \\
\hline & $\mathrm{R}_{\mathrm{bN} 1}$ & - & 4.67 & 3.69 & 2.14 & 4.43 & 5 & 4.1 & 3.36 & 2 & 2.33 & 5.33 \\
\hline & $\mathrm{R}_{\mathrm{bN} 2}$ & - & 3 & 4.32 & 7 & 3.5 & 4 & 5 & 3.12 & 2 & 3 & 3 \\
\hline & $\mathrm{R}_{\mathrm{bN} 3}$ & - & - & 4.17 & - & 2 & - & 4 & 8.50 & - & - & - \\
\hline & $\mathrm{R}_{\mathrm{bN} 4}$ & - & - & 6.00 & - & - & - & - & 2.00 & - & - & - \\
\hline & $\mathrm{R}_{\mathrm{bm}}$ & - & 1.9 & 4.5 & 2.3 & 2.5 & 2.3 & 3.3 & 4.2 & 1.0 & 1.3 & 2.1 \\
\hline & DdN1 & - & 2.01 & 1.44 & 1.91 & 1.42 & 1.86 & 1.63 & 1.76 & 0.94 & 1.43 & 1.27 \\
\hline & DdN2 & - & 1.18 & 0.58 & 0.84 & 3.1 & 0.59 & 0.83 & 0.72 & 1.16 & 0.35 & 0.71 \\
\hline & DdN3 & - & 0.16 & 0.28 & 0.6 & 0.6 & 0.45 & 0.11 & 0.36 & 0.45 & 0.66 & 0.33 \\
\hline & DdN4 & - & - & 0.23 & - & 0.17 & - & 0.38 & 0.05 & - & - & - \\
\hline & DdN5 & - & - & 0.11 & - & - & - & - & 0.41 & - & - & - \\
\hline
\end{tabular}


Table 2 (continued): Morphometric characteristics of the river network in the IGNP.

\begin{tabular}{|c|c|c|c|c|c|c|c|c|c|c|}
\hline \multicolumn{11}{|c|}{ Mountain range } \\
\hline \multicolumn{2}{|c|}{ Watershed } & 莺 & 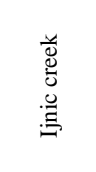 & 兽 & 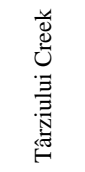 & 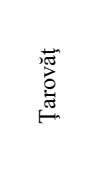 & 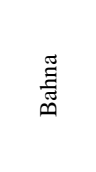 & $\frac{\pi}{\tilde{G}}$ & 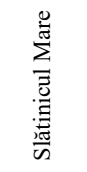 & 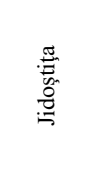 \\
\hline \multicolumn{2}{|c|}{$\begin{array}{c}\text { Danube } \\
\text { Confluence }\end{array}$} & $\mathrm{km}$ & $\begin{array}{l}\mathrm{km} \\
955\end{array}$ & $\begin{array}{l}\mathrm{km} \\
953\end{array}$ & $\begin{array}{c}\mathrm{km} \\
951.2\end{array}$ & $\begin{array}{l}\mathrm{km} \\
950\end{array}$ & $\begin{array}{l}\mathrm{km} \\
950\end{array}$ & $\begin{array}{l}\mathrm{km} \\
954\end{array}$ & $\begin{array}{l}\mathrm{km} \\
947\end{array}$ & $\begin{array}{c}\mathrm{km} \\
941.7\end{array}$ \\
\hline \multirow{12}{*}{ 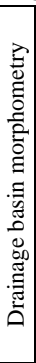 } & $\mathrm{A}$ & $\mathrm{km}^{2}$ & 1.5 & 3.7 & 1.4 & 15.18 & 137 & 16.26 & 7.38 & 20.87 \\
\hline & $\mathrm{P}$ & $\mathrm{km}$ & 6 & 8.3 & 0.52 & 21.2 & 73.92 & 20.14 & 12.8 & 22.21 \\
\hline & $\mathrm{L}$ & $\mathrm{km}$ & 2.18 & 2.48 & 1.48 & 8.44 & 23.97 & 7.22 & 4.06 & 7.1 \\
\hline & Lmax & $\mathrm{km}$ & 1.09 & 2.41 & 1.5 & 2.81 & 8.86 & 2.96 & 2.4 & 3.99 \\
\hline & $\mathrm{B}$ & $\mathrm{km}$ & 0.68 & 1.49 & 0.95 & 1.8 & 5.73 & 2.25 & 1.82 & 2.94 \\
\hline & $\mathrm{R}_{\mathrm{r}}$ & - & 0.13 & 0.17 & 0.15 & 0.08 & 0.04 & 0.08 & 0.12 & 0.06 \\
\hline & $\mathrm{R}_{\mathrm{f}}$ & - & 0.36 & 0.49 & 0.51 & 0.29 & 0.31 & 0.36 & 0.43 & 0.41 \\
\hline & $\mathrm{R}_{\mathrm{c}}$ & - & 0.52 & 0.67 & 65.03 & 0.42 & 0.31 & 0.50 & 0.57 & 0.53 \\
\hline & $\mathrm{S}_{\mathrm{b}}$ & $\%$ & 9.6 & 6.18 & 9.64 & 7.41 & 2.45 & 6.9 & 10.92 & 6.57 \\
\hline & $l_{\mathrm{v}}$ & $\mathrm{m}$ & 202 & 234 & 186 & 195 & 289 & 272 & 230 & 323 \\
\hline & Hmax & $\mathrm{m}$ & 346 & 480 & 288 & 719 & 1047 & 633 & 548 & 484 \\
\hline & Hmin & $\mathrm{m}$ & 64 & 64 & 67 & 80 & 62 & 62 & 71 & 34 \\
\hline \multirow{32}{*}{ 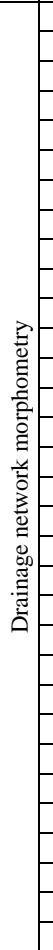 } & Lrâupp & $\mathrm{km}$ & 2.1 & 2.75 & 1.42 & 10.11 & 35 & 7.29 & 4.4 & 7.53 \\
\hline & $\sum \mathrm{L}$ & $\mathrm{km}$ & 4.09 & 8.69 & 4.13 & 42.78 & 261.09 & 32.89 & 17.68 & 35.56 \\
\hline & $\mathrm{D}_{\mathrm{d}}$ & $\mathrm{km} / \mathrm{km}^{2}$ & 2.73 & 2.35 & 2.95 & 2.82 & 1.91 & 2.02 & 2.4 & 1.7 \\
\hline & N1 & - & 7 & 10 & 7 & 53 & 285 & 32 & 25 & 35 \\
\hline & N2 & - & 2 & 2 & 2 & 13 & 89 & 7 & 8 & 10 \\
\hline & N3 & - & 1 & 1 & 1 & 5 & 21 & 2 & 1 & 1 \\
\hline & N4 & - & - & - & - & 1 & 4 & 1 & - & - \\
\hline & N5 & - & - & - & - & - & 1 & - & - & - \\
\hline & LmedN1 & $\mathrm{m}$ & 341.77 & 636.18 & 338.56 & 398.85 & 474.73 & 602.5 & 388.93 & 547.06 \\
\hline & LmedN2 & $\mathrm{m}$ & 282.25 & 294.3 & 647.25 & 695.57 & 593.14 & 667.84 & 620.71 & 937.19 \\
\hline & LmedN3 & $\mathrm{m}$ & 1137.81 & 1454.64 & 475.49 & 2001 & 1471.50 & 3513.5 & 3001 & 7084.71 \\
\hline & LmedN4 & $\mathrm{m}$ & - & - & - & 2598.2 & 5074.90 & 917.62 & - & - \\
\hline & LmedN5 & $\mathrm{m}$ & - & - & - & - & 21.893 & - & - & - \\
\hline & $\sum \mathrm{N} 1$ & $\mathrm{~km}$ & 2.39 & 6.36 & 2.36 & 21.14 & 135.21 & 19.27 & 9.72 & 19.11 \\
\hline & $\sum \mathrm{N} 2$ & $\mathrm{~km}$ & 0.564 & 0.883 & 1.29 & 9.042 & 52.79 & 4.67 & 4.96 & 9.37 \\
\hline & $\sum \mathrm{N} 3$ & $\mathrm{~km}$ & 1.137 & 1.45 & 0.47 & 10.005 & 30.90 & 7.02 & 3 & 7.084 \\
\hline & $\sum \mathrm{N} 4$ & $\mathrm{~km}$ & - & - & - & 2.598 & 20.30 & 0.917 & - & - \\
\hline & $\sum \mathrm{N} 5$ & $\mathrm{~km}$ & - & - & - & - & 21.89 & - & - & - \\
\hline & fN1 & - & 4.67 & 2.7 & 5 & 3.49 & 1.29 & 1.97 & 3.39 & 1.68 \\
\hline & $\mathrm{fN} 2$ & - & 1.33 & 0.54 & 1.43 & 0.86 & 0.40 & 0.43 & 1.08 & 0.48 \\
\hline & fN3 & - & 0.67 & 0.27 & 0.71 & 0.33 & 0.10 & 0.12 & 0.14 & 0.05 \\
\hline & fN4 & - & - & - & - & 0.07 & 0.02 & 0.06 & - & - \\
\hline & fN5 & - & - & - & - & - & - & - & - & - \\
\hline & $\mathrm{R}_{\mathrm{bN} 1}$ & - & 3.5 & 5 & 3.5 & 4.08 & 3.20 & 4.57 & 3.12 & 3.5 \\
\hline & $\mathrm{R}_{\mathrm{bN} 2}$ & - & 2 & 2 & 2 & 2.6 & 4.24 & 3.5 & 8 & 10 \\
\hline & $\mathrm{R}_{\mathrm{bN3}}$ & - & - & - & - & 5 & 5.25 & 2 & - & - \\
\hline & $\mathrm{R}_{\mathrm{bN} 4}$ & - & - & - & - & - & 4.00 & - & - & - \\
\hline & DdN1 & - & 1.59 & 1.72 & 1.69 & 1.39 & 0.61 & 1.19 & 1.32 & 0.92 \\
\hline & DdN2 & - & 0.38 & 0.24 & 0.92 & 0.6 & 0.24 & 0.29 & 0.67 & 0.45 \\
\hline & DdN3 & - & 0.76 & 0.39 & 0.34 & 0.66 & 0.14 & 0.43 & 0.41 & 0.34 \\
\hline & DdN4 & - & - & - & - & 0.17 & 0.09 & 0.06 & - & - \\
\hline & DdN5 & - & - & - & - & - & 0.10 & - & - & - \\
\hline
\end{tabular}




\section{RESULTS AND DISCUSSION}

The "Iron Gates" Natural Park hydrography is defined by the Danube River, which contributed significantly to the individualization of this unique region in Europe known as Danube Gorge, or the transversal valley of the Danube.

As a definitive element of the landscape the Danube River has an influence on the particularities of other landscape components. On the Romanian sector of the Danube Gorge, the river is supplied by a series of tributary rivers with springs in the Semenic Mountains, Almăj Mountains, Cernei Mountains and Mehedinți Mountains. These rivers are presented from West to East in figure 2 and listed in table 2.

Most of the watersheds are aligned on N-S or NW-SW directions. Radimna and Sirinea watersheds are exceptions to this general feature and their orientation is quite longitudinal in the park area on a direction E-W (Tetelea, 2005). The length of the perennial rivers increases from West to East. The river network type in the "Iron Gates" Natural Park is dendritic, and the tributary streams of the main rivers make a sharp angle at the confluence depending on the watershed lithology.

Watershed area is one of the most important morphometric parameters of a river basin, having a great influence on the water availability in the river bodies and in shaping their size as well as the number of stream within the river basin. The watersheds in Locvei Mountains have areas below $10 \mathrm{~km}^{2}$ with few exceptions of those watersheds extending outside the park limits in Locvei Mountains: Radimna $\left(\mathrm{A}=81.5 \mathrm{~km}^{2}\right)$, Valea Mare $(\mathrm{A}=$ $\left.25 \mathrm{~km}^{2}\right)$, Boşneag $\left(\mathrm{A}=55.6 \mathrm{~km}^{2}\right)$ and Gornea-Sicheviţa $\left(\mathrm{A}=83.8 \mathrm{~km}^{2}\right)$.

Watersheds in Almăj Mountains have areas larger than $10 \mathrm{~km}^{2}$ and it is reflected in the river hydrological conditions. The largest watershed areas are over $100 \mathrm{~km}^{2}$ and extend beyond the park limits: Oreviţa $\left(A=102 \mathrm{~km}^{2}\right)$, Berzasca $\left(A=229 \mathrm{~km}^{2}\right)$, Mraconia $\left(A=113 \mathrm{~km}^{2}\right)$. In Mehedinţi Mountains the seven watersheds that are inside the park have A between $1.4 \mathrm{~km}^{2}$ and $22.1 \mathrm{~km}^{2}$ with Bahna River basin extending outside the park limits with $\mathrm{A}=137 \mathrm{~km}^{2}$.

The length of the main rivers $\left(\mathrm{L}_{\text {râupp }}\right.$ ) from Locvei Mountains varies between $1.78 \mathrm{~km}$ (Popin Valley) and $32.5 \mathrm{~km}$ (Radimna) with a mean length of the main rivers of six $\mathrm{km}$ (Tab. 2). Most of the rivers run dry during the summer and have an intermittent character of torrentiality. The length of the main rivers in Almăj Mountains is between $1.13 \mathrm{~km}$ (Valea Mare) and $48.56 \mathrm{~km}$ (Berzasca) with a majority of the main rivers being longer than six $\mathrm{km}$. The majority of rivers from the eastern part of the "Iron Gates" Natural Park have length below $10 \mathrm{~km}$ with one exception of Bahna River $\mathrm{L}_{\text {râup }}=35 \mathrm{~km}$ which extends outside the park limit.

In the "Iron Gates" Natural Park are a series of small intermittent streams with small watersheds and a pronounced torrentiality hydrological pattern. These streams were not included in the morphometric analysis from table 2, and they are from West to East, Trăilă, Gârbovăț, Codicea Mică Stream, Călina Mică, Jorbăjului, Grigore, Dâlboca, Ivanului, Stamati Valley, Virului Valley, and Oglănic.

Stream size is an important component to water management and very important to geographers, geologists, hydrologists and other scientists because it gives them an idea of the size and strength of specific waterways within river networks. In addition, classifying stream order allows scientists to easily study the amount of sediment in an area and effectively use waterways as natural resources.

Stream order also helps in determining what types of life might be present in the waterway. This is the idea behind the River Continuum Concept (Vannote et al., 1980), a model used to determine the number and types of organisms present in a stream of a given 
size. Different types of plants for example can live in sediment filed, slower flowing rivers like the Danube than can live in a fast flowing tributary of the same river.

The highest stream order among the 63 watersheds is five and is shown by eight watersheds: Liuborajdea $\left(\mathrm{L}_{\text {medN5 }}=4.45 \mathrm{~km}\right)$, Gornea - Sichevița $\left(\mathrm{L}_{\text {medN5 }}=9.7 \mathrm{~km}\right)$, Orevița $\left(\mathrm{L}_{\text {medN5 }}=17.4 \mathrm{~km}\right)$, Berzeasca $\left(\mathrm{L}_{\text {medN5 }}=33.9 \mathrm{~km}\right)$, Sirinia $\left(\mathrm{L}_{\text {medN5 }}=17.2 \mathrm{~km}\right)$, Mraconia $\left(\mathrm{L}_{\text {medN5 }}=13.3 \mathrm{~km}\right)$, Eșelnița $\left(\mathrm{L}_{\text {medN5 }}=10 \mathrm{~km}\right)$ and Bahna $\left(\mathrm{L}_{\text {medN5 }}=21.8 \mathrm{~km}\right)$. Total number of stream or order five is $\mathrm{N}_{\mathrm{n}}=1$ for all these watersheds.

Form factor $\left(R_{f}\right)$ has values under one underlying the elongated shape of all watersheds within the "Iron Gates" Natural Park. The river basins with $\mathrm{R}_{\mathrm{f}}<0.4$ are considered very elongated (according to the classification of Diaconu and Lăzărescu, 1965), which is obvious in the park in the case of small streams and the rivers developed more longitudinally compared to their width. The river basins with $\mathrm{R}_{\mathrm{f}}$ between 0.4 and 0.5 are considered elongated ant those with $\mathrm{R}_{\mathrm{f}}>0.5$ are quasicircular (Mare Creek, Mraconia, Târziului, etc.). The large river basins with a well-developed drainage network and high values of the drainage density $\left(D_{d}\right)$ are not necessary elongated, make obvious the importance of lithology and relief in shaping the watersheds and explain the different values of $R_{f}$.

Relief ratio $\left(R_{r}\right)$ expresses the sediment load of the rivers (Hadley and Schum, 1961, cited by Gordon et al., 2004) and grows exponentially with relief ratio. The values of $R_{r}$ for the "Iron Gates" Natural Park watersheds are sub-unitary $R_{r}>1$ with the highest values of $R_{r}$ in the case of small stream watersheds with steep slopes: Vodenski and Mare creek $\left(R_{r}=0.24\right)$, Big Basin $\left(R_{r}=0.22\right)$, Dalbochi $\left(R_{r}=0.23\right)$, Bostița $\left(R_{r}=0.21\right)$. The values $R_{r}<1$ suggest a relatively high capacity of sediment load of these rivers, but without a continuous input of sediments in the Danube due to their intermittent character.

Mean slope of the watershed $\left(S_{b}\right)$ is correlated with drainage density and the relief of the watershed and has a strong influence on the runoff and sediment transport. The highest values of $S_{b}$ are found in Almăj Mountains $S_{b}=24.6$.

Bifurcation ratio $\left(R_{b}\right)$ does not remain constant from one order to the next because the variation in lithology and geometry but it tends to be constant throughout the series. High $\mathrm{R}_{\mathrm{bm}}$ indicates early hydrograph peak with a potential of flash flooding during spring. Higher $\mathrm{R}_{\mathrm{bm}}$ values are the characteristics of structurally more disturbed watersheds with prominent distortion in drainage pattern and vice versa (Nag, 1998). Maximum $\mathrm{R}_{\mathrm{bm}}$ is seen in several watersheds, Berzeasca $\left(\mathrm{R}_{\mathrm{bm}}=4.8\right)$, Mraconia $\left(\mathrm{R}_{\mathrm{bm}}=4.5\right)$, Eșelnița $\left(\mathrm{R}_{\mathrm{bm}}=4.2\right)$, Morilor Stream $\left(\mathrm{R}_{\mathrm{bm}}=4.1\right)$. Here earlier hydrograph peaks are expected indicating strong structural control on the drainage development for these watersheds.

Based on the River Continuum Concept perspective (Vannote et al., 1980), it is assumed that high values of $\mathrm{R}_{\mathrm{b}}$ suggests a larger contribution of a higher number of streams of inferior order with organic matter to high order streams. The total and mean length of low stream orders tributaries, together with the drainage density, have a contribution to the uptake and transport potential of organic matter and dissolved matter of rivers. We assume that watersheds with developed upstream river network and with high number of inferior stream orders and considerable lengths contribute with a higher amount of organic matters to the trophic circuit of the river systems. However, such assumptions are needed to verify the importance of morphometric elements in the development of the river systems with primary productivity ratios calculations or quantitative distribution or different groups of organisms in the river systems (Tetelea, 2005). 
Stream frequency $\left(f N_{n}\right)$ indicator shows the contribution of different stream orders with the amount of organic matter. The low values of $\mathrm{fN}_{\mathrm{n}}$ indicate a slow discharge time and longer peak times of each of these stream orders because of low runoff rates due to fewer streams draining the watersheds. Further, it is noted that $\mathrm{fN}_{\mathrm{n}}$ decreases as the stream number increases.

Drainage density $\left(D_{d}\right)$ controls the travel time of the water within a river basin (Patton, 1998). Generally, a low value of $D_{d}$ suggests areas with highly resistant permeable material and vegetative cover and low relief. High drainage density is noted in areas of week and permeable subsurface material and sparse vegetation and mountainous relief. Examination of $D_{d}$ values from table 2 indicates that most of the watersheds have low $D_{d}$ values (below two $\left.\mathrm{km} / \mathrm{km}^{2}\right)$. Watersheds with moderate $D_{\mathrm{d}}$ values $\left(2.0-2.5 \mathrm{~km} / \mathrm{km}^{2}\right)$ are found in all mountains from the "Iron Gates" Natural Park, like Popin Valley, Silianschi, Big Valley - Divici, Valea Adâncă, Susca, Crușovița, Reșița Mare, etc. Dalbochi, Plavișevița, Mraconia, Sohodol, Tarovat, Ijnic, Târziului watersheds have high $D_{d}$ values (between 2.5 and $3.0 \mathrm{~km} / \mathrm{km}^{2}$ ). Values of $D_{d}$ higher than three $\mathrm{km} / \mathrm{km}^{2}$ are found in Eșelnița, Mala, Costeneț and Strineacului. On the basis of low $D_{d}$ value it is assumed that these watersheds will have the greatest basin lag time while the watersheds with high $\mathrm{D}_{\mathrm{d}}$ value will demonstrate the shortest lag time. From the geological point of view low and moderate $D_{d}$ values reveal a composition of watersheds with permeable subsurface material, good vegetation cover and low relief, which results in more infiltration capacity and water recharge.

Circularity ratio $\left(R_{c}\right)$ is the ratio of the area of the basin to the area of circle having the same circumference as the basin perimeter (Miller, 1953). This parameter is influenced by the length and frequency of the streams, geological structures, land use and land cove, climate, relief and slope of the basin. Low values of $R_{c}$ indicate low relief and impermeable subsurface resulting in lower basin lag time and these watersheds will show shorter time to peak flow. On the opposite high $R_{c}$ values characterize high relief, elongated and permeable surface resulting in greater basin lag time and these watersheds will show delayed time to peak.

The mountainous character of the Danube's tributary rivers is also shown by their longitudinal profiles in figure 3. This character is obvious from spring to pouring mouth and has an influence on the hydrological regime throughout the feeding and flow variation, flow velocity, turbidity, etc. The rivers from the "Iron Gates" Natural Park are typical to the Western Carpathian types and are characterized by high waters during spring and winter. Winter flow is high due to the sub-Mediterranean climatic influences that contribute to melting of the snow. The feeding of the rivers is done more than 50\% through surface waters coming from rain and snow. Some of the small rivers that run through karst substrate are fed by underground springs. The lithology has a big influence on river hydrology, especially on big rivers like Cerna and Nera where due to diversity of lithology and area of the watershed, the hydrologic regime is fluctuating according to the geological area.

The water discharge of the rivers in the "Iron Gates" Natural Park is influenced by the quantity of precipitation available at the watershed. Maximum discharge is recorded usually at the end of winter and the beginning of spring (February-April), when the early snow melt due to warm Mediterranean air overlap with the spring rains. This is the beginning of the high waters of spring. From April on a period of continuous decrease of water runoff dictates the considerable low discharge of rivers and even water depletion of small streams, which run downstream into own sediments. July-September is the driest period of the year and some of the rivers run dry due to the sub-Mediterranean climate and low frequency of rain. 


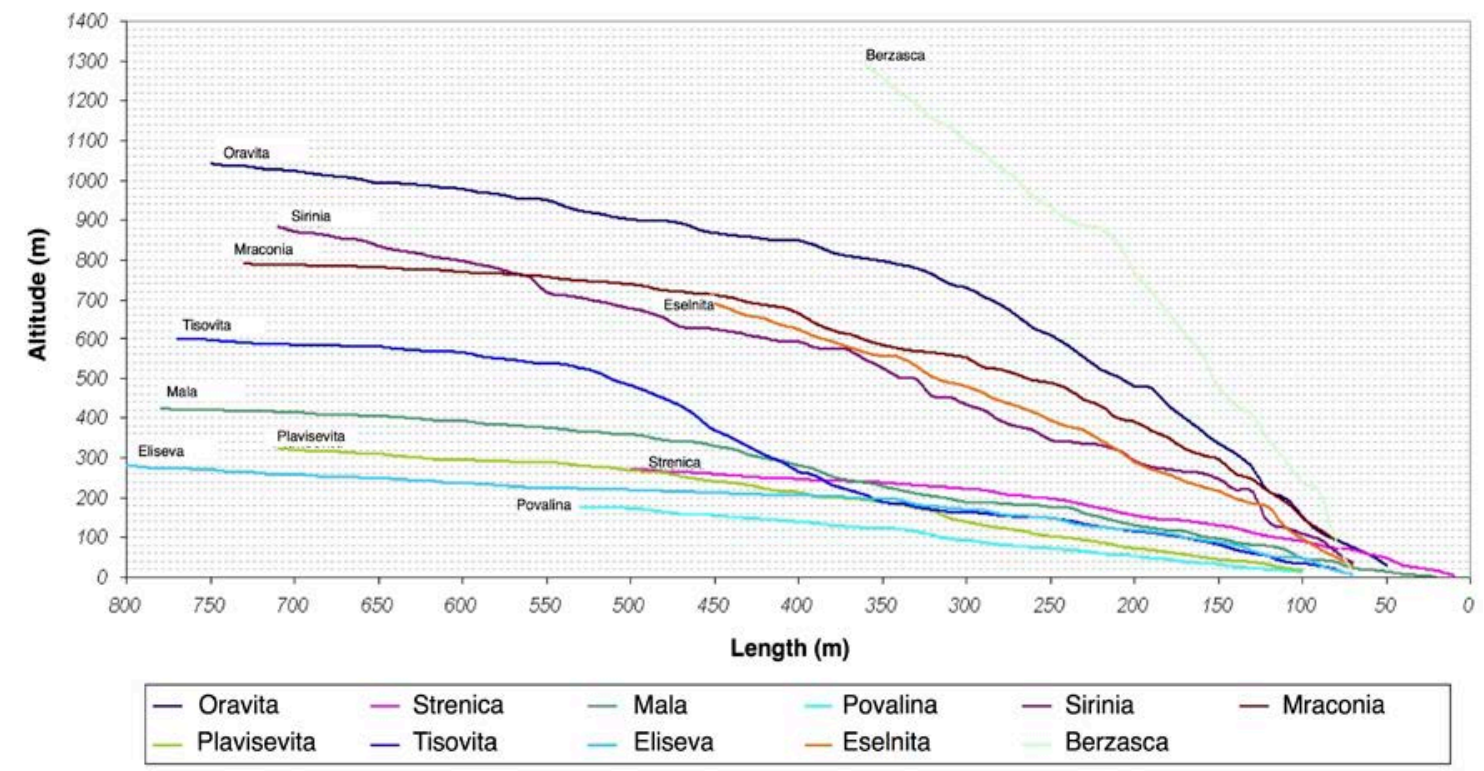

Figure 4: Longitudinal profiles of the main trybutaries to the Danube from Almăj Mountains.

From this point of view there are three river categories in the "Iron Gates" Natural Park: perennial streams and rivers that never run dry even in the most torrid summer, like Cerna, Nera, Berzasca, Bahna; semi-perennial streams and rivers, which run dry only in the most arid years, like Vodiţa, Dubova, Cozla, Mala, etc.; intermittent streams and rivers, which run dry every year during the summer, like Ribiş, Reciţa, Cameniţa, Glaucina and creeks.

After their mean multiannual discharge, the Danube's tributary rivers from the "Iron Gates” Natural Park can be classified as: large mountainous rivers, with watershed area between 200 and $1,500 \mathrm{~km}^{2}$, length of the river between 30 and $100 \mathrm{~km}$ and mean discharge between 0.5 and $15 \mathrm{~m}^{3} / \mathrm{s}$. Berzasca and Cerna falls into this category; small mountainous rivers, with watershed area between 100 and $200 \mathrm{~km}^{2}$, length of the river between 20 and $50 \mathrm{~km}$ and mean discharge between 0.2 and $5 \mathrm{~m}^{3} / \mathrm{s}$. Eșelnița, Mraconia, Sirinia, Bahna and other rivers are in this category; mountainous streams, with much smaller watershed areas, river lengths and discharge that of the small mountainous rivers. This category is including most of the tributary streams to the Danube in the "Iron Gates" Natural Park: Dubova, Liubotina, Plavişeviţa, Reciţa Mare, Povalina, Iuţi, Elişeva, Oreviţa, Liuborajdea, etc.

Solid discharge is conditioned by four factors: morphometry of the relief, structure of lithology, watershed cover (grass, vegetation, etc.) and discharge frequency. In the "Iron Gates" Natural Park the complex lithological structure of volcanic, sedimentary and metamorphic rocks defines the sediment components, which are mostly gravel and boulders. Dragged sediments predominate against suspended sediments. The narrow river valleys confined by abrupt versants and steep slopes, the riverbed has large boulders with a diameter of $1 \mathrm{~m}$ to $1.5 \mathrm{~m}$ that are covered with a rich vegetative layer.

The quantity of eroded and transported material by the rivers into the Danube has been changed since the water level has risen due to the damming of the river. Because of this the relief energy decreases with almost $50 \mathrm{~m}$ and the decreased transport capacity of the rivers, and the aggradation of sediments shifted to upstream. This contributed greatly to the filling with 
sediments of the lower segments of the tributary rivers to the Danube. The gulfs along the confluence of the rivers with the Danube started to form submersible deltas which will grow in the future with the input of more sediment from the Danube and upstream (Figs. 5a, b).
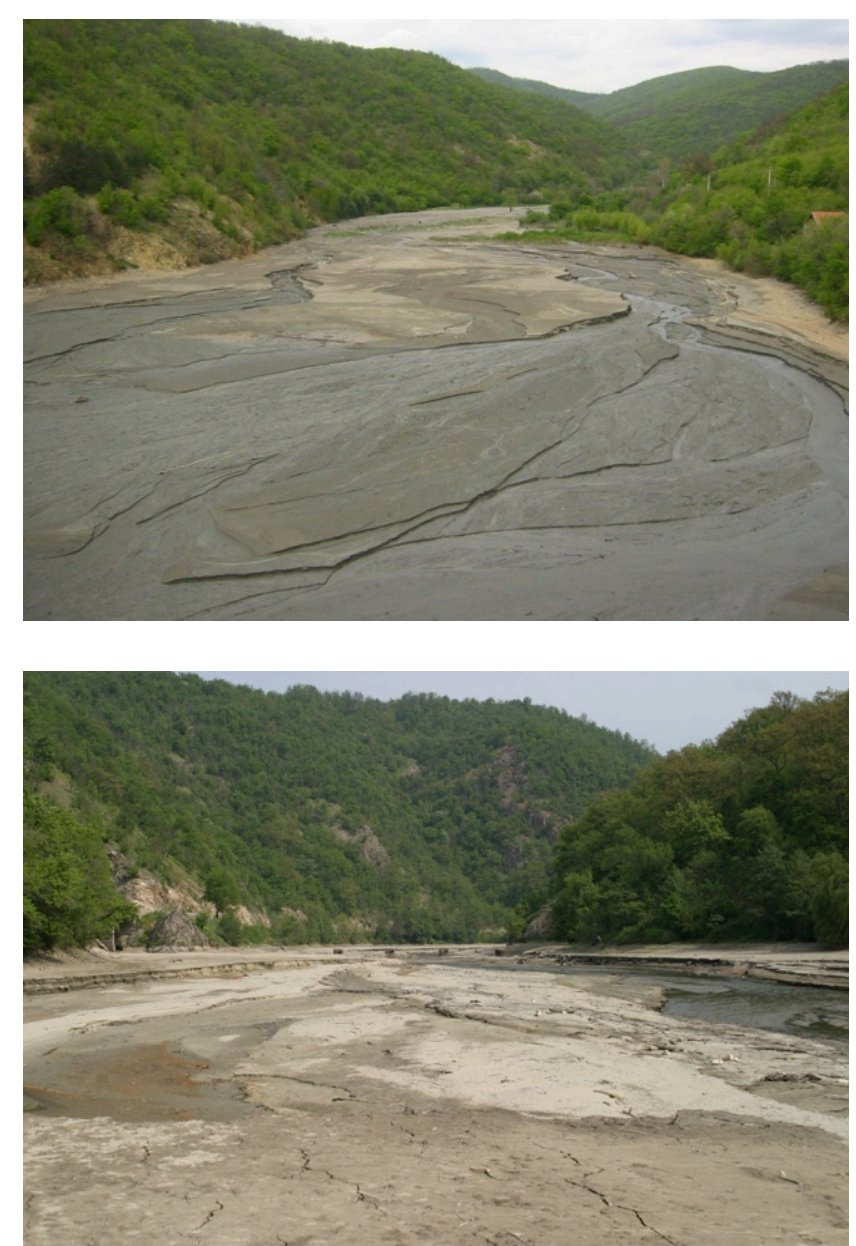

Figure 5a, b: Alluvial processes with the formation of submerse deltas in Bahna gulf (left) and Vodiţa (right).

\section{CONCLUSIONS}

The forming elements of the landscape and their role in the dynamics and geoecological potential of running waters are determined by a hierarchical control of processes and interactions within a geosystem. This control can be shown through morphometric analysis of watersheds. The identification of watershed geomorphology and physical processes forms the basis for understanding the spatial extent of the riparian forest as well as stream habitats, their quality.

The morphometric parameters of the river network with relevance to the determination of geoecological potential of river ecosystems in the "Iron Gates" Natural Park are the total number of stream segments of the same order, total and mean length of stream segments of an order, bifurcation ratio, and circularity ratio. A positive aspect for the good status of the rivers in the "Iron Gates" Natural Park is the distribution and frequency of the human settlements and 
the existing quarries in the watersheds of the Danube's tributary rivers. These are concentrated especially along the high order streams in the lower part of the watersheds especially on the confluence with the Danube River.

Using the morphometric analysis of the river network and watersheds can be of great support in the determination of river ecosystem health. However, the evaluation of river habitats in the "Iron Gates" Natural Park and the changes imposed by the potential loses of their specific structure and functions should be a continuous research theme for the future. The results of this study can be useful for the conservation of aquatic and riparian habitats; as well as, for the ecological restoration of certain river segments and water bodies.

The evaluation of the aquatic habitats loss and their components remains an open question since the expansion of build areas, arable land use, and spill of untreated sewage waters in the park area was not addressed enough by the existing researches.

\section{ACKNOWLEDGEMENTS}

Author is thankful to Mrs. Pătroescu M., University of Bucharest for the inspiration and continuous support in pursuing this study.

\section{REFERENCES}

1. Farrukh A., Gowhar M. and Shakil A. R., 2013 - Morphometric Analysis to Infer Hydrological Behaviour of Lidder Watershed, Western Himalaya, India, Geography Journal, http://dx.doi.org/10.1155/2013/178021 (accessed in 27 May 2015).

2. Curtean A., Morariu A., Făcălău S., Bianca L. and Chişu S., 1999 - Data concerning the benthic communities of the Cibin River (Olt River Basin), Transylvanian Review of Systematical and Ecological Research, The Upper and Middle Olt River Basin, 1, 99-110.

3. Gordon D. N., McMahon A. T., Finlayson L. B., Gippel J. C. and Nathan J. R., 2004 - Stream Hydrology - An introduction for Ecologists, Second Edition, Wiley, 429.

4. Manko P., 2008 - Aquatic macroinvertebrate communities in River Torysa across the Javrina military training area in Levočske Vrchy Mountains (Slovakia), Transylvanian Review of Systematical and Ecological Research, The Wetlands Diversity, 6, 41-50.

5. Miller V. C., 1953 - A Quantitative geomorphic study of drainage basin characteristics in the Clinch Mountain area, Virginia and Tennessee, Technical Report, 3, 389-402, Columbia University, Department of Geology, ONR, New York, NY, USA, 1953.

6. Nag S. K., 1998 - Morphometric analysis using remote sensing techniques in the Chaka subbasin, West Bengal, Journal of Indian Society of Remote Sensing, 26, 1-2, 69-76.

7. Naiman J. R. and Bilby E. R., 2001 - River Ecology and Management, Springer, 705.

8. Patton P. C., 1998 - Drainage basin morphometry and floods, Flood Geomorphology, 51-64, John Wiley and Sons, New York, USA, 1988, 51-64.

9. Tetelea C., 2005 - Potențialul geoecologic al ecosistemelor acvatice din Parcul Natural Porțile de Fier, Teză de doctorat, Universitatea din București, 232. (in Romanian)

10. Trichkova T., Stefanov T., Vassilev M. and Zivkov M., 2009 - Fish species diversity in the rivers of the north-west Bulgaria, Transylvanian Review of Systematical and Ecologica Research, The Wetlands Diversity, 8, 161-168.

11. Troll C., 1969/1971 - Landscape ecology and biogeoecology, Geoforum, 8, 43-46.

12. Vannote L. R., Minshall G. W., Cummins K. W., Sedell J. R. and Cushing C. E., 1980 - The river continuum project, Canadian Jorunal of Fisheries and Aquatic Sciences, 37, 130-137.

13. Zăvoianu I., 1978 - Morfometria bazinelor, Edit. Academiei R. S. R., Bucureşti. (in Romanian)

14. * I. N. M. H., 1994 - Instrucţiuni metodologice pentru calculul scurgerii maxime în bazine mici. (in Romanian) 